\title{
Global wellposedness of a free boundary problem for the navier-stokes equations in an exterior domain
}

\begin{abstract}
In this paper, we prove a unique existence theorem of globally in time strong solutions to free boundary problem for the Navier-Stokes equations in an exterior domain in the case that initial data are small enough. The key step is to prove decay properties of locally in time solutions, which is derived by combination of maximal $\mathrm{L}_{\mathrm{p}}-\mathrm{L}_{\mathrm{q}}$ regularity with $\mathrm{L}_{\mathrm{p}}-\mathrm{L}_{\mathrm{q}}$ decay estimates for solutions of slightly perturbed Stokes equations with free boundary condition in an exterior domain.
\end{abstract}

Keywords: navier-stokes equations, free boundary problem, global well-posedness, exterior domain, polynomial decay, maximal $l_{p}-l_{q}$ regularity
Volume I Issue 2 - 2017

\author{
Yoshihiro Shibata \\ Department of Mathematics and Research Institute of Science \\ and Engineering,Waseda University, Japan
}

\author{
Correspondence: Yoshihiro Shibata, Department of \\ Mathematics and Research Institute of Science and Engineering, \\ Waseda University, Ohkubo 3-4-I, Shinjuku-ku, Tokyo 169-8555, \\ Japan, Email yshibata325@gmail.com
}

Received: May 29, 2017| Published: October, 17, 2017

\section{Introduction}

This paper deals with global well-posedness of the following free boundary problem for the Navier-Stokes equations. Let $\Omega$ be an exterior domain in the $N$ dimensional Eucledian space $\mathrm{R}^{N}$ occupied by an incompressible viscous fluid. Let $\Gamma$ be the boundary of $\Omega$ that is a $C^{2}$ compact hyper surface with the unit outer normal n. Let $\Omega_{t}$ be the evolution of $\Omega$ at time $t$. Let $\Gamma_{t}$ be the boundary of $\Omega_{t}$ with the unit outer normal $\mathrm{n}_{t}$. Let $\rho$ and $\mu$ be positive numbers denoting the mass density and the viscosity coefficient, respectively. Let $u={ }^{T}\left(u_{1}, \ldots, u_{N}\right)$ be an $N$-vector of functions describing the velocity field, where ${ }^{T} M$ denotes the the transposed $M$, and let $\mathrm{p}$ be a scalar function describing the pressure field. We consider the initial boundary value problem for the Navier-Stokes equations in $\Omega$ given by

$$
\begin{array}{clc}
\rho(\partial t \mathbf{u}+\mathbf{u} \cdot \nabla \mathbf{u})-\operatorname{Div}(\mu \mathbf{D}(\mathbf{u})-\mathbf{P I})=0, & \operatorname{div} \mathbf{u}=0 & \text { in } \bigcup_{0<t<T} \Omega_{t} \times\{t\}, \\
(\mu \mathbf{D}(\mathbf{u})-\mathbf{P I}) \mathbf{n}_{t}=0, & V_{\Gamma t}=\mathbf{n}_{t} \cdot \mathbf{u} & \text { on } \bigcup_{0<t<T} \Gamma_{t} \times\{t\}, \\
\left.\mathbf{u}\right|_{t=0}=\mathbf{u}_{0},\left.\Omega_{t}\right|_{t=0}=\Omega &
\end{array}
$$

Here, $D(u)=\nabla u+{ }^{\cdot} \nabla u$ denotes the doubled deformation tensor, I the $N \times N$ identity matrix, and $V_{\Gamma t}$ the evolution speed of the surface $\Gamma_{t}$ in the $\mathrm{n}_{t}$ direction. Moreover, for any matrix field $\mathrm{K}$ with $(i, j)$ component $\mathrm{K}_{\mathrm{ij},}$ the quantity $\mathrm{Div} \mathrm{K}$ is an $N$ vector of functions whose $\mathrm{i}^{\text {th }}$ component is $\sum_{j=1}^{N} \partial_{j} K_{i j}, \partial_{j}=\partial / \partial x_{j}$ and for any $N$ vector of function $\mathbf{w}=\left(w_{1}, \ldots, w_{N}\right), \operatorname{div} \mathbf{w}=\sum_{j=1}^{N} \partial_{j} w_{i}$ and the quantity $\mathbf{w} \cdot \nabla \mathbf{w}$ is an $N$-vector of functions whose $\mathrm{i}^{\text {th }}$ component is $\sum_{j=1}^{N} \partial_{j} w_{i}$ One phase problem for the Navier-Stokes equations formulated in (1) with $(\mu D(u)-\mathrm{P} I) n t=c_{\sigma} H n_{t}$ in place of $(\mu D(u)-\mathrm{P} I) n t=0$ has been received wide attention for many years, where $H$ is the doubled mean curvature of $\Gamma_{t}$ and $c$ is a non-negative constant describing the coefficient of surface ${ }^{\sigma}$ tension. In particular, the following two cases have been studied by many mathematicians: (1) the motion of an isolated liquid mass and (2) the motion of a viscous incompressible fluid contained in an ocean of infinite extent. In case (1), the initial domain $\Omega$ is bounded and local well posedness in the case that $c \sigma>0$ was proved by Solonnikov ${ }^{1-4}$ in the $L_{2}$ SobolevSlobodetskii space, by Schweizer ${ }^{5}$ in the semi group setting, and by Moglievskii et al., ${ }^{6}$ in the Holder spaces. And, in the case that $c \sigma=0$, local wellposedness was proved by Solonnikov, ${ }^{7}$ Mucha et al., ${ }^{8,9}$ in the $L_{p}$ Sobolev-Slobodetskii space, and by Shibata ${ }^{10,11}$ in the $L_{p}$ in time and $L_{q}$ in space setting. Global wellposedness in the case that $c \sigma=0$ for small initial data by Solonnikov ${ }^{4,7}$ in the $L_{p}$ SobolevSlobodetskii space and by Shibata ${ }^{12}$ in the $L_{p}$ in time and $L_{q}^{p}$ in space setting. Global wellposedness in the case that $c \sigma=0$ was proved under the assumption that the initial domain $\Omega$ is sufficiently close to a ball and initial data are very small by Solonnikov ${ }^{13}$ in the $L_{2}$ SobolevSlobodetskii space, by Padula et al., ${ }^{14}$ in the Holder spaces, and by Shibata ${ }^{15}$ in the $L_{p}$ in time and $L_{q}$ in space setting.

In case (2), the initial domain $\Omega_{0}$ is a perturbed layer like:

$$
\Omega_{0}=\left\{x \in \mathbb{R}^{3} \mid-b<x_{3}<\eta\left(x^{\prime}\right), x^{\prime}=\left(x_{1}, x_{2}\right) \in \mathbb{R}^{2}\right\} \text { and }
$$

local wellposed was proved by Allain, ${ }^{16}$ Beale $^{17}$ and Tani ${ }^{18}$ in the $L_{2}$ Sobolev-Slobodetskii space when $c \sigma>0$ and by Abels ${ }^{19}$ in the $L_{p}$ Sobolev-Slobodetskii space when $c \sigma=0$.

Global wellposedness for small initial velocity was proved in the $L_{2}$ Sobolev-Slobodetskii space by Beale ${ }^{20}$ and Tani et al., ${ }^{21}$ in the case that $c \sigma>0$ and by Sylvester ${ }^{22}$ in the case that $c \sigma=0$ . The decay rate was studied by Beale et al., ${ }^{23}$ Sylvestre, ${ }^{24}$ Hataya $^{25}$ $\&$ Hataya et al. $^{26}$ In the case of the Ocean problem without bottom, $\Omega_{0}=\left\{x \in \mathbb{R}^{3} \mid x_{3}<\eta\left(x^{\prime}\right), x^{\prime}=\left(x_{1}, x_{2}\right) \in \mathbb{R}^{2}\right\}$.

In this case, global well posedness for small initial data and the decay properties of solutions have been studied by Saito et al. ${ }^{27-29}$ Recently, local well-posedness for the one phase problem of the Navie-Stokes equations was proved in the general unbounded domain case by Shibata ${ }^{12}$ in the $c \sigma=0$ case and by Shibata ${ }^{29,30}$ in the case $c \sigma>0$.

We remark that two phase problem of the Navier-Stokes equations 
has been studied by many math- ematicians, ${ }^{31-46}$ and references therein. Although many papers dealt with global well-posend, as far as the author knows, global well-posedness of free boundary problem for the Navier-Stokes equations in an exterior domain has never be treated, and the purpose of this paper is to prove global wellposedness of problem (1) in the $L_{p}$ in time and $L_{q}$ in space setting. Since only polynomial decays are obtained for solutions of Stokes equations with free boundary conditions in the exterior domain case; ${ }^{47,48}$ it is necessary to choose a large exponent $p$ to guarantees $L_{p}$ integrability of solutions, so that the maximal $L_{p}-L_{q}$ regularity for the Stokes equations with free boundary consition proved in Shibata ${ }^{30,49,50}$ and also in Pruess et al., ${ }^{40}$ in the different $p$ and $q$ case are one of essential tools.

Now we consider the transformation that transforms $\Omega_{t}$ to a fixed domain. If $\Omega$ is a bounded domain, then we have the exponential stability of the corresponding Stokes equations with free boundary conditions in some quotient space, so that we can use the Lagrange transformation to transform $\Omega_{t}$ to $\Omega .^{7,12}$ But, is now an exterior domain, so that solutions of the Stokes equations with free boundary conditions decay polynomially as mentioned above. Thus, the Lagrange transformation is not available, because the polynomial decay does notoseem to be enough to control the term $\int_{0}^{t} \nabla u d s$ . Another known transformation is the Hanzawa one. But, this transformation requires at least the $W_{q}^{3-1 / q}(N<q)$ regularity of the height function representing $\Gamma$, and such regularity is usually derived from surface tension. In our case, surface tension is not taken into account, so that such regularity is unable to be obtained. To overcome such difficulty, our idea is to use the Lagrange transformation only near $\Gamma$.

Let $R$ be a positive number such that $\mathrm{O}=\mathbb{R}^{N} \backslash \Omega \subset B_{R / 2}$, where $B_{L}=\left\{x \in \mathbb{R}^{N}|| x \mid<L\right\}$, and let $\kappa$ be $a C^{\infty}$ function such that $\kappa(\xi)=1$ for $|\xi| \leq R$ and $\kappa(\xi)=0$ for $|\xi| \geq 2 R$. Let $\mathbf{v}(\xi, t)$ and $q(\xi, t)$

be the velocity field and the pressure field in Lagrange coordinates

$\{\xi\}$. Let us consider a transformation,

$$
x=\mathbf{L}(\xi, t):=\xi+\int_{0}^{t} \kappa(\xi) \mathbf{v}(\xi, s) d s
$$

Let $\delta$ be a positive number such that the transformation: $x=\mathbf{L}(\xi, t)$ is bijective from $\Omega$ on to $\Omega_{t}=\{x=\mathbf{L}(\xi, t) \mid \xi \in \Omega\}$ for each $t \in(0, T)$ provided that

$$
\int_{0}^{T}\|\nabla(\kappa(\cdot) \mathbf{v}(\cdot, s))\| L_{\infty}(\Omega) d s \leq \delta
$$

Since $\delta$ will be chosen as a small positive number eventually, we may assume that $0<\delta \leq 1$. Let

$$
\ell_{i j}(t)=\delta_{i j}+\int_{0}^{t} \frac{\partial}{\partial \xi_{j}}\left(\kappa(\xi) v_{i}(\xi, s)\right) d s\left(\mathbf{v}=\cdot\left(v_{1}=, \ldots, v_{N}\right)\right), \mathbf{A}(t)\left(\ell_{i j}(t)\right)^{-1}=\left(a_{i j}(t)\right)
$$

where $\delta_{i j}$ are the Kronecker delta symbols, that is $\delta_{i i}=1$ and $\delta_{i j}=0$ for $i \neq j$. Here and hereafter, a function $a=a(\xi, t)$ is written simply by $a(t)$ and $\left(a_{i j}\right)$ denotes the $N \times N$ matrix whose $(i, j)$ component is $a_{i j}$, unless confusion may occur. For a while, we assume that the $N \times N$ matrix $\left(\ell_{i j}(t)\right)$ is invertible.

Let

$$
\begin{aligned}
& \Omega_{t}=\{x=\mathbf{L}(\xi, t) \mid \xi \in \Omega\}, \quad \Gamma_{t}=\{x=\mathbf{L}(\xi, t) \mid \xi \in \Gamma\}, \\
& \mathbf{u}(x, t)=\mathbf{v}\left(\mathbf{L}^{-1}(x, t), t\right) \text { and } p(x, t)=q\left(\mathbf{L}^{-1}(x, t), t\right) \text { in Eq. }
\end{aligned}
$$

and then, $\mathbf{v}$ and $q$ satisfy the following

equations:

$$
\left\{\begin{array}{rc}
\partial_{t} \mathbf{v}-\operatorname{Div}(\mu \mathbf{D}(\mathbf{v})-q \mathbf{I})=\mathbf{f}(\mathbf{v}), & \text { in } \Omega \times(0, T), \\
\operatorname{div} \mathbf{v}=g(\mathbf{v})=\operatorname{div} \mathbf{g}(\mathbf{v}) & \text { in } \Omega \times(0, T), \\
(\mu \mathbf{D}(\mathbf{v})-q \mathbf{I}) \mathbf{n}_{5} \mathbf{h}(\mathbf{v}) & \text { on } \Gamma \times(0, T), \\
\left.\mathbf{v}\right|_{t=0}=\mathbf{u}_{0} & \text { in } \Omega .
\end{array}\right.
$$

Here, $f(v)$ is consisting of some linear combinations of nonlinear functions of the forms

$V_{0}\left(\int_{0}^{t} \nabla(\kappa \mathbf{v}) d s\right) \nabla^{2} \mathbf{v}, V_{1}\left(\int_{0}^{t} \nabla(\kappa \mathbf{v}) d s\right) \partial_{t} \mathbf{v}$

$V_{2}\left(\int_{0}^{t} \nabla(\kappa \mathbf{v}) d s\right)\left(\int_{0}^{t} \nabla^{2}(\kappa \mathbf{v}) d s\right) \nabla \mathbf{v},(1-\kappa)\left({ }^{\cdot} \mathbf{A}\right)^{-1}\left\{\mathbf{v} \cdot\left({ }^{\cdot} \mathbf{A} \nabla \mathbf{v}\right)\right\} ;$

and $g(\mathrm{v})$ and $g(\mathrm{v})$ and $\mathrm{h}(\mathrm{v})$ are nonlinear functions of the forms:

$g(\mathbf{v})=V_{3}\left(\int_{0}^{t} \nabla(\kappa \mathbf{v}) d s\right) \nabla \mathbf{v} ; \mathbf{g}(\mathbf{v})=V_{4}\left(\int_{0}^{t} \nabla(\kappa \mathbf{v}) d s\right) \mathbf{v} ; \mathbf{h}(\mathbf{v})=V_{5}\left(\int_{0}^{t} \nabla(\kappa \mathbf{v}) d s\right) \nabla \mathbf{v}$ $=2$.

with some nonlinear functions $V_{i}$ such that $V_{i}(0)=0$ except for $i$

The main result of this paper is the following theorem that shows the unique existence theorem of global in time solutions of Eq. (5) and asymptotics as $t \rightarrow \infty$.

\section{Theorem |}

Let $N \geq 3$ and let $\mathrm{q}_{1}$ and $\mathrm{q}_{2}$ be exponents such that $\max \left(N, \frac{2 N}{N-2}\right)<\mathrm{q}_{2}<\infty$ and $1 / q_{1}=1 / q_{2}+1 / N$. Let $\mathrm{b}$ and $\mathrm{p}$ be numbers defined by

$$
b=\frac{3 N}{2 q_{2}}+\frac{1}{2}, p=\frac{2 q_{2}(1+\sigma)}{q_{2}-N}
$$

with some very small positive number $\sigma$. Then, there exists an $\in>0$ such that if initial data $u_{0} \in B_{q_{2, p}}^{2(1-1 / p)}(\Omega)^{N} \cap B_{q_{1 / 2}, p}^{2(1-1 / p)}(\Omega)^{N}$ satisfies the compatibility condition:

$$
\operatorname{div} \mathrm{u}_{0}=0 \text { in } \Omega, \mathbf{D}\left(\mathbf{u}_{0}\right) \mathbf{n}-<\mathbf{D}\left(\mathbf{u}_{0}\right) \mathbf{n}, \mathbf{n}>\mathbf{n}=0 \text { on } \Gamma
$$

and the smallness condition: with $I=\left\|\mathbf{u}_{0}\right\| B_{q_{2}, p}^{2(1-1 / p)}+\left\|\mathbf{u}_{0}\right\| B_{q_{12}, p}^{2(1-1 / p)}$, then Eq. (5) admits unique solutions $\mathrm{v}$ and $\mathrm{q}$ with $\mathbf{v} \in L_{p}\left((0, \infty), H_{q_{2}}^{2}(\Omega)^{N}\right) \cap H_{p}^{1}\left((0, \infty), L_{q_{2}}(\Omega)^{N}\right), \quad q \in L_{p}\left((0, \infty), H_{q_{2}}^{1}(\Omega)+\hat{H}_{q_{2}, 0}^{1}(\Omega)\right)$ possessing the estimate $[v]_{\infty} \leq C \epsilon$ with 


$$
\begin{aligned}
& {[\mathbf{v}]_{T}=\left\{\int_{0}^{T}\left(\langle s\rangle^{b}\|\mathbf{v}(\cdot, s)\| H_{\infty}^{1}(\Omega)\right)^{p} d s\right.} \\
& \left.+\int_{0}^{T}\left(\langle s\rangle=\left(\mathrm{b}-\frac{N}{2 q_{1}}\right)\|\mathbf{v}(\cdot, s)\| H_{q_{1}}^{1}(\Omega)\right)^{p} d s+\sup _{0<s<T}\langle s\rangle^{\frac{N}{2 q_{1}}}\|\mathbf{v}(\cdot, s)\| L_{q 1}(\Omega)\right)^{p} \\
& \left.+\int_{0}^{T}\left(\langle s\rangle^{\left(\mathrm{b}-\frac{N}{2 q 2}\right)}\|\mathbf{v}(\cdot, s)\| H_{q 2}^{2}(\Omega)+\left\|\partial_{t} \mathbf{v}(\cdot, s)\right\| L_{q 2}(\Omega)\right)^{p} d s\right\}^{\frac{1}{p}} .
\end{aligned}
$$
of $\epsilon$.

Here, $\left\langle s>=\left(1+s^{2}\right)^{1 / 2}\right.$ and $\mathrm{C}$ is a constant that is independent

\section{Remark 2}

Let $1 / p^{\prime}=1-1 / p$. that is $1 / p^{\prime}=1-1 / p$. And then,

$$
\frac{1}{p^{\prime}}=\frac{(1+2 \sigma) q_{2}+N}{2 q_{2}(1+\sigma)}
$$

We choose $\sigma>0$ small enough in such a way that the following relations hold,

$$
\begin{aligned}
& 1<q_{1}<2, \frac{N}{q_{1}}>b>\frac{1}{p^{\prime}},\left(\frac{N}{q_{1}}-b\right) p>1,\left(\mathrm{~b}-\frac{N}{2 q_{2}}\right) \mathrm{p}>1, b \geq \frac{N}{2 q_{1}}, \\
& b \geq \frac{N}{q_{2}},\left(\frac{11) N}{2 q_{2}}+\frac{1}{2}\right) p^{\prime}<1,\left(\mathrm{~b}-\frac{N}{2 q_{2}}\right) p^{\prime}>1, \frac{N}{q_{2}}+\frac{2}{p}<1 .
\end{aligned}
$$

\section{Remark 3}

The exponent $\mathrm{q}_{2}$ is used to control the nonlinear terms, so that $\mathrm{q}_{2}$ is chosen as $N<q_{2}<\infty$.

Let

$$
\frac{1}{q_{1}}=\frac{1}{N}+\frac{1}{q_{2}}, \frac{1}{q_{3}}=\frac{1}{q_{1}}+\frac{1}{q_{2}}
$$

And the condition: $\mathrm{q}_{2}>\frac{2 N}{N-2}$ implies that $\mathrm{q}_{1}>2$ and $\mathrm{q}_{3}>1$ which is necessary to prove Theorem 1 .

Thus, we assume that

\section{Remark 4}

$$
\max \left(N, \frac{2 N}{N-2}\right)<q_{2}<\infty
$$

We can choose $\delta>0$ so small that $\mathrm{x}=\mathrm{L}(\xi, \mathrm{t})$ is a diffeomorphism with suitable regularity from $\Omega$ onto $\Omega t$, so that the original problem (1) is globally well-posed.

Finally, we explain several symbols used in this paper. We use bold small letters to denote $\mathrm{N}$-vectors of functions and bold capital letters to denote $\mathrm{N} \times \mathrm{N}$ matrix of functions. For a scalor function $\mathrm{h}=\mathrm{h}(\mathrm{x})$, $\nabla h$ is an $\mathrm{N}$ vector whose $\mathrm{i}^{\text {th }}$ component is $\partial_{i} h=\partial h / \partial x_{i}$. For $\mathrm{v}(\mathrm{x})$ $=\left(\mathrm{v}_{1}(\mathrm{x}), \ldots, \mathrm{v}_{\mathrm{N}}(\mathrm{x})\right), \nabla v$ is an $\mathrm{N} \times \mathrm{N}$ matrix of functions whose (i, j) component is $\partial_{i} v_{j}$. Given exponent $1<q<\infty$, let $\mathrm{q}^{\prime}=\mathrm{q} /(\mathrm{q}-1)$.

Let $L_{q}(\Omega), H_{q}^{m}(\Omega)$ and $B_{q, p}^{s}(\Omega)$ be the standard Lebesgue, Sobolev, and Besov spaces on $\Omega$, while $\|\bullet\| L_{q}(\Omega),\|\bullet\| H_{q}^{m}(\Omega)$, $\|\bullet\| B_{q, p}^{s}(\Omega)$ denote their norms, respectively. For a Banach space $\mathrm{X}$ with norm $\|\bullet\| X$,

Let $\quad\left\{\left(f_{1}, \ldots, f_{d}\right) \mid f_{i} \in X(i=1, \ldots, d)\right\}, \quad$ while the norm of $\mathrm{X}_{\mathrm{d}}$ is written simply by $\|\bullet\| X$, which is defined by $\|f\| X=\sum_{j=1}^{d}\left\|f_{j}\right\| X$ for $\quad f=\left(f_{1}, \ldots, f_{d}\right) \in X_{d}$. Let $\hat{H}_{q, 0}^{1}(\Omega)=\left\{\theta \in L_{q, l o c}(\Omega)\left|\nabla \theta \in L_{q}(\Omega)^{N}, \theta\right| \Gamma=0\right\}$

$$
\hat{H}_{q}^{1}(\Omega)=\hat{H}_{q, 0}^{1}(\Omega)+\left\{\mathfrak{p}=\mathfrak{p}_{1}+\mathfrak{p}_{2} \mid \mathfrak{p}_{1} \in H_{q}^{1}(\Omega), \mathfrak{p}_{2} \in \hat{H}_{q, 0}^{1}(\Omega)\right\}
$$

For $1 \leq p \leq \infty, L_{p}((a, b), X)$ and $H_{m}^{p}((a, b), X)$ denote the standard Lebesgue and Sobolev spaces of

$\mathrm{X}$-valued functions defined on an interval (a, b), while $\|\bullet\| H_{m}^{p}((a, b), X)$ and $\|\bullet\| H_{m}^{p}((a, b), X)$ denote their norms, respectively. The letter $\mathrm{C}$ denotes generic constants and $\mathrm{C}_{\mathrm{a}, \mathrm{b}, \mathrm{c}}, \ldots$ means that the constant $\mathrm{C}_{\mathrm{a}, \mathrm{b}, \mathrm{c},} \ldots$ depends on $\mathrm{a}, \mathrm{b}, \mathrm{c} \ldots$. The values of $\mathrm{C}$ and $\mathrm{C}_{\mathrm{a}, \mathrm{b}, \mathrm{c}}$ may change from line to line.

After Introduction (Sect. 1), the paper is organized as follows: In Sect. 2, we reformulate problem (5) by using the formula:

$$
\int_{0}^{t} \nabla\left(\kappa(\xi) \mathbf{v}(\xi, s) d s=\int_{0}^{T} \nabla\left(\kappa(\xi) \mathbf{v}(\xi, s) d s-\int_{0}^{T} \nabla(\kappa(\xi) \mathbf{v}(\xi, s) d s .\right.\right.
$$

In Sect. 3, we give estimations of the nonlinear terms. In Sect. 4 , we explain how to prolong local in time solutions to the infinite time interval $(0, \infty)$. Finally, in Sect. 5 , we prove Theorem 1 by using maximal $L_{p}-L_{q}$ regularity and $L_{p}-L_{q}$ decay estimate for solutions of the perturbed Stokes equations with free boundary condition in an exterior domain, which was proved in Shibata. ${ }^{30,47}$

\section{Another formulation of Eq. (5)}

Let $T>0$ and let

$$
\mathbf{v} \in H_{p}^{1}\left((0, T), L_{q}(\Omega)^{N}\right) \cap L_{p}\left((0, T), H_{q}^{2}(\Omega)\right), q \in L_{p}\left((0, T), H_{q}^{1}(\Omega)+\hat{H}_{q, 0}^{1}(\Omega)\right)
$$

be solutions of Eq. (5) satisfying the condition (3). In what follows, we rewrite Eq. (5) in order that the nonlinear terms have suitable decay properties.

Let $\mathbf{A}(t)=\left(a_{i j}(t)\right)$ be the $\mathrm{N} \times \mathrm{N}$ matrix given in (4), and let $\mathbf{n}_{t}=\cdot\left(n_{t 1}, \ldots, n_{t N}\right)$ and $\mathbf{n}=\cdot\left(n_{1}, \ldots, n_{N}\right)$, and then by the transformation (2), we have

$$
a_{\mathrm{ji}}(\mathrm{t}) \frac{\partial}{\partial \xi_{j}}, \frac{\partial}{\partial x_{j}}=\sum_{j=1}^{N} n_{\mathrm{ti}}=d(\mathrm{t}) \sum_{j=1}^{N} a_{\mathrm{ji}}(\mathrm{t}) n_{j}
$$


where $d(\mathrm{t})=|T \mathbf{A}(t) \mathbf{n}|$.

$$
a_{j i}(\mathrm{t})=\delta_{i j}+\tilde{a}_{i j}(t), J(t)=1+\tilde{J}(t), \ell_{i j}(t)=\delta_{i j}+\tilde{\ell}_{i j}(t),
$$

Where $\ell_{i j}$ are given in (4) and $J$ is the Jacobian of the transformation (2), that is $J=J=\operatorname{det}\left(\ell_{i j}\right)$, and then

$\tilde{a}_{i j}(t)=b_{i j}\left(\int_{0}^{t} \nabla(\kappa(\xi) \mathbf{v}(\xi, s)) d s\right), \tilde{J}(t)=K\left(\int_{0}^{t} \nabla(\kappa(\xi) \mathbf{v}(\xi, s)) d s\right)$, $\left.\tilde{\ell}_{i j}(t)=m_{i j}\left(\int_{0}^{t} \nabla(\kappa(\xi) \mathbf{v}(\xi, s)) d s\right):=\int_{0}^{t} \frac{\partial}{\partial \xi_{j}}\left(\kappa(\xi) \mathbf{v}_{i}(\xi, t)\right) d s\right)$

With some smooth functions $b_{i j}$ and $K$ defined on $\{\mathbf{w}|| \mathbf{w} \mid \leq 1\}$ such that $b_{i j}(0)=K(0)=0$, where $\mathbf{w}$ is the corresponding variable to $\int_{0}^{t} \nabla(\kappa(\xi) \mathbf{v}(\xi, s)) d s$ Let $\mathbf{u}(x, t)=\mathbf{v}(\xi, t)$ and $p(x, t)=q(\xi, t)$ and then $\mathrm{u}$ and $\mathrm{p}$ satisfy Eq. (1). By (14),

$$
\frac{\partial u_{i}}{\partial x_{j}}+\frac{\partial u_{j}}{\partial x_{i}}=D_{i j, t}(\mathrm{v}):=D_{i j}(\mathrm{v})+\tilde{D}_{i j}(t) \Delta \mathrm{v}
$$

With

$$
D_{i j}(\mathrm{v})=\frac{\partial v_{i}}{\partial \xi_{j}}+\frac{\partial v_{j}}{\partial \xi_{j}}, \quad \tilde{D}_{i j}(t) \Delta \mathrm{v}=\sum_{K=1}^{N} \tilde{a}_{k j}(t) \frac{\partial v_{i}}{\partial \xi_{k}}+\tilde{a}_{k i}(t) \frac{\partial v_{j}}{\partial \xi_{k}}
$$

We also have an important formula:

$$
\operatorname{div} \mathbf{u}=\sum_{j=1}^{N} \frac{\partial u_{j}}{\partial x_{j}(18), j, k=1} \sum^{N} J(t) a_{k j}(t) \frac{\partial v_{j}}{\partial \xi_{k}}=\sum_{j, k=1}^{N} \frac{\partial}{\partial \xi_{k}}\left(J(t) a_{k j}(t) v_{j}\right)
$$

which implies that

$$
\sum_{j, k=1}^{N} \tilde{a}_{k j}(t)=\tilde{J}(t) a_{k j}(t) \frac{\partial v_{j}}{\partial \xi_{k}}=\sum_{j, k=1}^{N} \frac{\partial}{\partial \xi_{k}}\left\{\left(\tilde{a}_{k j}(t)+\tilde{J}(t) a_{k j}(t)\right) v_{j}\right\}
$$

And then, Eq. (5) is written as follows:

$$
\left\{\begin{aligned}
\sum_{i=1}^{N} \ell_{i s}(t)\left(\partial_{t} v_{i}+(1-\kappa) \sum_{j, k=1}^{N} v_{j} a_{k j}(t) \frac{\partial v_{i}}{\partial \xi_{k}}\right) & \\
-\mu \sum_{i, j, k=1}^{N} \ell_{i s}(t) a_{k j}(t) \frac{\partial}{\partial \xi_{k}} D_{i, j, t}(\mathrm{v})-\frac{\partial q}{\partial \xi_{s}}=0 & \text { in } \Omega \times(0, \mathrm{~T}), \\
\sum_{j, k=1}^{N} J(t) a_{k j}(t) \frac{\partial v_{j}}{\partial \xi_{k}}=\sum_{j, k=1}^{N} \frac{\partial}{\partial \xi_{k}}\left(J(t) a_{k j}(t) v_{j}\right)=0 & \text { in } \Omega \times(0, \mathrm{~T}) \\
(20) \quad \sum_{i, j, k=1}^{N} \ell_{i s}(t) a_{k j}(t) D_{i, j, t}(\mathrm{v}) n_{k}-q n_{s}=0 & \text { on } \Gamma \times(0, \mathrm{~T}), \\
\left.\mathrm{v}\right|_{t=0}=\mathbf{u}_{0} & \text { in } \Omega
\end{aligned}\right.
$$

Where $s$ runs from 1 through $N$. Here, we have used the fact that $\left(\ell_{i j}\right)=\mathrm{A}^{-1}$ which follows from (4).

In order to get some decay properties of the nonlinear terms, we write

$\int_{0}^{t} \nabla\left(\kappa(\xi) \mathbf{v}(\xi, s) d s=\int_{0}^{T} \nabla\left(\kappa(\xi) \mathbf{v}(\xi, s) d s-\int_{0}^{T} \nabla(\kappa(\xi) \mathbf{v}(\xi, s) d s\right.\right.$.
In (16), by the Taylor formula we write

$$
\begin{array}{ll}
a_{i j}(t)=a_{i j}(T)+A_{i j}(t), & \ell_{i j}(t)=\ell_{i j}(T)+L_{i j}(t), \\
D_{i j, t}=D_{i j, T}(\mathrm{v})+D_{i j}(t) \Delta \mathrm{v} & J(t)=J(T)+J(\mathrm{t})
\end{array}
$$

With

$A_{i j}(t)=\int_{0}^{1}-b_{i j}^{\prime} \int_{0}^{T} \nabla\left(\kappa(\xi) \mathbf{v}(\xi, s) d s-\theta \int_{t}^{T} \nabla(\kappa(\xi) \mathbf{v}(\xi, s) d s) d \theta \int_{t}^{T} \nabla(\kappa(\xi) \mathbf{v}(\xi, s) d s\right.$, $L_{i j}(t)=-\int_{t}^{T} \frac{\partial}{\partial \xi_{j}} \kappa(\xi) v_{i}(\xi, s) d s, \quad D_{i j}(t) \Delta v=\sum_{\kappa=1}^{N}\left(A_{k j}(t) \frac{\partial v_{i}}{\partial \xi_{k}}+A_{k i} \frac{\partial v_{j}}{\partial \xi_{k}}\right)$,

$J(\mathrm{t})=-\int_{0}^{1} K^{\prime}\left(\int_{0}^{T} \nabla\left(\kappa(\xi) \mathbf{v}(\xi, s) d s-\theta \int_{t}^{T} \nabla(\kappa(\xi) \mathbf{v}(\xi, s) d s) d \theta \int_{t}^{T} \nabla(\kappa(\xi) \mathbf{v}(\xi, s) d s\right.\right.$

Where $b_{i j}^{\prime}$ and $K^{\prime}$ are derivatives of $b_{i j}$ and $K$ with respect to w. By the relation:

$$
\sum_{s=1}^{N} \ell_{i s}(T) a_{s m}(T)=\delta_{i m},
$$

the first equation in (20) is rewritten as follows:

$$
\partial_{t} v_{m}-\mu \sum_{j, k=1}^{N} a_{j k}(T) \frac{\partial}{\partial \xi_{k}}\left(\mu D_{m j, \mathrm{~T}}(\mathrm{v})-\delta_{m j} q=f_{m}(\mathrm{v})\right.
$$

With

$$
\begin{aligned}
f_{m}(\mathrm{v})= & -\sum_{s=1}^{N} a_{s m}(T)\left\{\sum_{i=1}^{N} L_{i s}(t) \partial_{t} v_{i}+\sum_{i, j, k=1}^{N}(1-\kappa) \ell_{i s}(t) a_{k j}(t) v_{i} \frac{\partial v_{i}}{\partial \xi_{k}}\right\} \\
& +\mu \sum_{k}^{N} a_{s m}(T)\left\{\sum_{i, j, k=1}^{N} \ell_{i s}(T) a_{k j}(T) \frac{\partial}{\partial \xi_{k}}\left(D_{i j}(\mathrm{t}) \nabla \mathrm{v}\right)+\sum_{i, j, k=1}^{N} \ell_{i s}(T) A_{k j}(t) \frac{\partial}{\partial \xi_{k}} D_{i j, \mathrm{t}}(\mathrm{v})\right\} \\
& \left(23^{\prime 3}\right) \\
& +\sum_{i, j, k=1}^{N} L_{i s}(t) a_{k j}(t) \frac{\partial}{\partial \xi_{k}} D_{i j, \mathrm{t}}(\mathrm{v})
\end{aligned}
$$

Next, by (18)

$$
\widetilde{\operatorname{div} v}=g(v)=\operatorname{div} g(v)
$$

With

$$
\begin{aligned}
& \widetilde{d i v} v=\sum_{j, k=1}^{N} J(T) a_{k j}(T) \frac{\partial v_{i}}{\partial \xi_{k}}=\sum_{j, k=1}^{N} \frac{\partial}{\partial \xi_{k}} J(T) a_{k j}(T) \mathrm{v}_{j} \\
& \mathrm{~g}(\mathrm{v})=\sum_{(24)^{k=1}}^{N}\left(J(T) A_{k j}(t)+J(t) a_{k j}(t)\right) \frac{\partial v_{j}}{\partial \xi_{k}} \\
& \mathrm{~g}_{k}(\mathrm{v})=\sum_{j=1}^{N}\left(J(T) A_{k j}(t)+J(t) a_{k j}(t) \mathrm{v}_{j}, \mathrm{~g}(\mathrm{v})=\left(\mathrm{g}_{1}(\mathrm{v}) \ldots . \mathrm{g}_{\mathrm{N}}(\mathrm{v})\right)\right.
\end{aligned}
$$

Finally, we consider the boundary condition. Let $\tilde{\mathbf{n}}$ be an $\mathrm{N}$-vector defined on $\mathbb{R}^{N}$ such that $\tilde{\mathbf{n}}=\mathbf{n}$ on $\Gamma$ and $\|\tilde{\mathbf{n}}\| H_{\infty}^{2}\left(\mathbb{R}^{N}\right) \leq C$. In what follows, $\tilde{\mathbf{n}}$ is simply written by $n=\left(n_{1}, \ldots, n_{N}\right)$. By (14) and (22)

$$
\sum_{j, k=1}^{N} a_{j k}(T)\left(\mu D_{m j, \mathrm{~T}}(\mathrm{v})-\delta_{m j} q\right) n_{k}=h_{m}(\mathrm{v})
$$

with 
$h_{m}(\mathrm{v})=-\mu \sum_{j, k=1}^{N}\left(a_{k j}(T) D_{m j}(t) \nabla \mathrm{v}+A_{k j}(t) D_{m j, t}(\mathrm{v})\right) n_{k}-\mu \sum_{i, j, k, s=1}^{N} a_{s m}(T) L_{i s}(t) a_{k j}(t) D_{i j, t}(v) n_{k}$

By (18),

$\mu \sum_{j, k=1}^{N} a_{k j}(T) \frac{\partial}{\partial \xi_{k}}\left(\mu D_{m j, \mathrm{~T}}(\mathrm{v})-\delta_{m j} q\right)=J(T)^{-1} \sum_{j, k=1}^{N} \frac{\partial}{\partial \xi_{k}}\left\{J(T) a_{j k}(T)\left(D_{m j, \mathrm{~T}}(\mathrm{v}) \delta_{m j} q\right)\right\}$

And

$\sum_{j, k=1}^{N} a_{k j}(T) D_{m j, T}(\mathrm{v}) n_{k}-\left(\sum_{s=1}^{N} a_{s m}(T) n_{s}\right) q=\sum_{k=1}^{N}\left[\sum_{j=1}^{N}\left\{J(T) a_{k j}(T)\left(D_{m j, T}(\mathrm{v})-\delta_{m j} q\right\}\right] n_{k}\right.$

Thus, letting

$S_{m k}(\mathbf{v}, q)=\sum_{j=1}^{N} J(T) a_{k j}(T)\left(\mu D_{m j, T}(\mathbf{v})-\delta_{m j} q\right), \quad \tilde{\mathbf{S}}(\mathbf{v}, q)=\left(S_{i j}(\mathbf{v}, q)\right)$, $\mathrm{f}(\mathrm{v})=^{\cdot}\left(f_{1}(\mathrm{v}), \ldots, f_{N}(\mathrm{v})\right), \quad h(\mathrm{v})=\cdot\left(h_{1}(\mathrm{v}), \ldots, h_{N}(\mathrm{v})\right)$,

and using (18), we see that $\mathrm{v}$ and $\mathrm{q}$ satisfy the following equations:

$$
\left\{\begin{aligned}
\partial_{t} \mathrm{v}-J(T)^{-1} D i v \tilde{S}(\mathrm{v}, q)=f(\mathrm{v}) & \text { in } \Omega \times(0, \mathrm{~T}), \\
\widetilde{\operatorname{div} v}=g(v)=\operatorname{div} g(v) & \text { in } \Omega \times(0, \mathrm{~T}), \\
\tilde{\mathbf{S}}(\mathbf{v}, q) \mathrm{n}=\mathrm{h}(\mathrm{v}) & \text { on } \Gamma \times(0, \mathrm{~T}), \\
\left.\mathrm{v}\right|_{t=0}=\mathrm{u}_{0} & \text { in } \Omega .
\end{aligned}\right.
$$

\section{Estimates for the nonlinear terms}

Let $\mathrm{f}(\mathrm{v}), \mathrm{g}(\mathrm{v})$, and $\mathrm{h}(\mathrm{v})$ are functions defined in Sect. 2. In this section, we estimate these functions. In what follows we write

$\left\|<t>^{\alpha} \mathbf{w}\right\| L_{p}((0, T), X)=\left\{\int_{0}^{T}\left(<t>^{\alpha}\|\mathbf{w}(\cdot, t)\| X\right)^{p} d t\right\}^{1 / p} 1 \leq p<\infty$, $\left\|<t>^{\alpha} \mathbf{w}\right\| L_{\infty}((0, T), X)=\underset{0<t<T}{\operatorname{esssup}}<t>^{\alpha}\|\mathbf{w}(\cdot, t)\| X \quad p=\infty$.

First, we prove that

$\|<t\rangle^{b} \mathbf{f} \| L_{p}\left((0, T), L_{q 1 / 2}(\Omega)+\|<t>\mathbf{f}\| L_{p}\left((0, T), L_{q_{2}}(\Omega)\right) \leq C\left(I+[\mathbf{v}]_{T}^{2}\right)\right.$.

with $\quad I=\left\|\mathrm{u}_{0}\right\|_{B_{q 1 / 2}^{2(1-p)}(\Omega)}+\left\|\mathrm{u}_{0}\right\|_{B_{q_{2}, p}^{2(1-1 / p)}(\Omega)}$. Here and in what follows, $\mathrm{C}$ denotes generic constants independent of $I,[v] T, \delta$, and $T$. The value of $\mathrm{C}$ may change from line to line. Since we choose $I$ small enough eventually, we may assume that $0<I \leq 1$. Especially, we use the estimates:

$$
I^{2} \leq I, \quad I[\mathbf{v}]_{T} \leq \frac{1}{2}\left(I^{2}+[\mathbf{v}]_{T}^{2}\right) \leq I+[\mathbf{v}]_{T}^{2}
$$

Since

$\int_{\alpha}^{\beta}\|\nabla(\kappa \mathbf{v}(\cdot, s))\| L_{\infty}(\Omega) \leq C(1+\alpha)^{-b+\frac{1}{p^{\prime}}}\left(\int_{\alpha}^{\beta}\left(\left\langle s>^{b}\|\mathbf{v}(\cdot, s)\| H_{\infty}^{1}(\Omega)\right)^{p} d s\right)^{1 / p}\right.$

$\int_{\alpha}^{\beta}\left\|\nabla^{2}(\kappa \mathbf{v}(\cdot, s))\right\| L_{q}(\Omega) \leq C(1+\alpha)^{-b+\frac{N}{2 q_{2}}+\frac{1}{p^{\prime}}}\left(\int_{\alpha}^{\beta}\left(\left\langle s>{ }^{b-\frac{N}{2 q_{2}}}\|\mathbf{v}(\cdot, s)\| H_{q_{2}}^{2}(\Omega)\right)^{p} d s\right)^{1 / p}\right.$ for any $0 \leq \alpha<\beta \leq T$, where $q \in\left[1, q_{2}\right]$, we have

$$
\begin{aligned}
& \int_{\alpha}^{\beta}\|\nabla(\kappa \mathbf{v}(\cdot, s))\| L_{\infty}(\Omega) d s \leq C[\mathbf{v}]_{T}(1+\alpha)^{-b+\frac{1}{p^{\prime}}} \\
& \int_{\alpha}^{\beta}\left\|\nabla^{2}(\underset{\kappa}{(28)}(\cdot, s))\right\| L_{q}(\Omega) \leq C[\mathbf{v}]_{T}
\end{aligned}
$$

for any $0 \leq \alpha<\beta \leq T$, where $q \in[1, q]$, because $b>\frac{N}{2 q_{2}}+\frac{1}{p^{\prime}}$ as follows from (11). By real interpolation

theorem, we have

$\sup _{t \in(0, T)}\left\langle t>^{b-\frac{N}{2 q_{2}}}\|\mathbf{v}(\cdot, t)\|_{B_{q_{2}, p}^{2(1-1 / p)}(\Omega)} \leq C\left\|\mathrm{u}_{0}\right\|_{B_{q_{2}, p}^{2(1-1 / p)}(\Omega)}\right.$

$+\left\|<t>{ }^{(29) \frac{)^{N}}{2 q_{2}}} \mathrm{v}\right\|_{L_{p}\left((0, T), H_{q_{2}}^{2}(\Omega)\right)}+\left\|<t>^{b-\frac{N}{2 q_{2}}} \partial t \mathrm{v}\right\| L_{p}\left((0, T) \mathrm{L}_{\mathrm{q}_{2}}(\Omega)\right)$

To prove (29), we introduce an operator $T(t)$ acting of $g \in B_{q_{2}, p}^{2(1-1 / p)} \mathbb{R}^{N}$ defined by

$$
T(t) g=F^{-1}\left[e^{-(|\xi|+1) t} F[g](\xi)\right],
$$

where $F$ and $F^{-1}$ denote the Fourier transform in $\mathbb{R}^{N}$ and its inverse transform. We have

$e_{(3 t)}^{\gamma t} T(t) g\left\|_{L\left((0, \infty), H_{q}^{2}\left(\mathbb{R}^{N}\right)\right)}+e^{\gamma t} \partial t T(t) g\right\|_{L p\left((0, \infty), L q\left(\mathbb{R}^{N}\right)\right)} \leq C\|g\|_{\substack{B^{2(1-1 / p)}\left(\mathbb{R}^{N}\right) \\ q_{2}, p}}$

Given $f(\mathrm{t})$ defned on $(0, \mathrm{~T})$ with $\left.f\right|_{t=0}=0$, let

$$
\left[e_{T} f\right](t)= \begin{cases}0 & (t<0), \\ f(t) & (0<t<T), \\ f(2 T-t) & (T<t<2 T), \\ 0 & (2 T<t),\end{cases}
$$

and then $\left[e_{T} f\right](t)=f(t)$ for $t \in(0, T)$ and

$$
\partial t\left[e_{T} f\right](t)= \begin{cases}0 & (t<0), \\ \partial t f(t) & (0<t<T), \\ -(\partial t f)(2 T-t) & (T<t<2 T), \\ 0 & (2 T<t),\end{cases}
$$

Let $\tilde{u}_{0}$ be an N-vector of functions in $B_{q_{2}, p}^{2(1-1 / p)}\left(\mathbb{R}^{N}\right)^{N}$ such that $\tilde{u}_{0}=u_{0}$ in $\Omega$ and $\left\|\tilde{\mathrm{u}}_{0}\right\|_{B_{q 1 / 2}^{2}, p} \mathbb{R}^{N}<C\left\|\mathrm{u}_{0}\right\|_{B_{q_{2}, p}^{2(1-1 / p)}(\Omega)}$.

L e t $z=e_{T}\left[<t>^{b-\frac{N}{2 q_{2}}} \mathrm{v}-T(|\mathrm{t}|) \tilde{\mathrm{u}}_{0}\right]+T(|\mathrm{t}|) \tilde{\mathrm{u}}_{0}$ for $t \in \mathbb{R}$.

Since $\langle t\rangle^{b-\frac{N}{2 q_{2}}} \mathrm{v}-\left.T(|\mathrm{t}|) \tilde{\mathrm{u}}_{0}\right|_{t=0}=0$ in $\Omega$ by (31), (32) and (33), 
$\|\mathbf{z}\|_{L_{p}\left((0, \infty), H_{q_{2}}^{2}(\Omega)\right)}+\left\|\partial_{t} \mathbf{z}\right\|_{L_{p}\left((0, \infty), L_{q_{2}}(\Omega)\right)}$

$\left\langle C\left(\left\|<t>{ }^{b-\frac{N}{2 q_{2}}} \mathrm{v}\right\| L_{p}\left((0, T), H_{q_{2}}^{2}(\Omega)\right)+\left\langle t>^{b-\frac{N}{2 q_{2}}} \partial_{t}\left\|_{\|}\right\|_{p}\left((0, T) \mathrm{L}_{\mathrm{q}_{2}}(\Omega)\right)+\left\|\mathrm{u}_{0}\right\|_{B_{q_{2}, p}(1-1 / p)}(\Omega)\right.\right.\right.$

Itisknown(Tanabe $)^{51}$ that $L_{p}\left((0, \infty), E_{1}\right) \cap H_{p}^{1}\left((0, \infty), E_{0}\right)$ is continuously imbedded into $\operatorname{BUC}\left((0, \infty),\left(E_{0}, E_{1}\right)_{1-1 / p, p}\right)$, where $E_{0}$ and $E_{1}$ are two Banach spaces such that $E_{1}$ is a dense subset of $\mathrm{E}_{0}$, and $\mathrm{BUC}$ denotes the set of all uniformly bounded continuous functions . Noting that

$$
z(t)=\left\langle t>^{b-\frac{N}{2 q_{2}}} v(t) \text { for } t \in(0, T)\right. \text {, we have }
$$

$$
\sup _{0<t<T)}\left\langle t>^{b-\frac{N}{2 q_{2}}}\|\mathbf{v}(t)\|_{B_{q_{2}, p}^{2(1-1 / p)}(\Omega)} \leq \sup _{t \in(0, \infty)}\|\mathrm{z}(t)\|_{B_{q_{2}, p}^{2(1-1 / p)}(\Omega)} \leq C\|\mathrm{z}\|_{L p\left((0, \infty), H_{q_{2}}^{2}(\Omega)\right)}+\left\|\partial_{t} z\right\|_{L_{p}\left((0, \infty), \mathrm{L}_{\mathrm{q}_{2}}(\Omega)\right)}\right.
$$

which, combined with (34), furnishes (29).

Since $2 / p+N / q_{2}<1, B_{q_{2}, p}^{2(1-1 / p)}(\Omega)$ is continuously imbedded into $H_{\infty}^{1}(\Omega)$ so that by (29)

$$
\left\langle t>^{b-\frac{N}{2 q_{2}}}\|\mathbf{v}(t)\|_{L \infty\left((0, \mathrm{~T}), H_{\infty}^{1}(\Omega)\right)} \leq C\left(I+[\mathrm{v}]_{T}\right)\right.
$$
Applying (3), (28) and (29) to the formulas in (15) and (16) and using the fact that $-b+\frac{1}{p^{\prime}} \frac{N}{2 q_{2}} \leq-\frac{N}{2 q_{2}}$ and $-b+\frac{N}{2 q_{2}} \leq-\frac{N}{2 q_{2}}$, which
follows from (11), give

$$
\begin{gathered}
\|\left(a_{i j}(t), J(t), \ell_{i j}(t), A_{i j}(t), J(\mathrm{t}), L_{i j}(t) \|_{L_{\infty}(\Omega)} \leq C,\right. \\
\|\left(A_{i j}(t), J(\mathrm{t}), L_{i j}(t)\left\|_{L_{\infty}(\Omega)} \leq C \int_{t}^{T} \nabla(\kappa \mathbf{v}(\cdot, s))\right\|_{L_{\infty}}(\Omega) d s \leq C[\mathrm{v}]_{T}+<t>^{-b+\frac{1}{p^{\prime}}} \leq C[\mathrm{v}]_{T}<t>>^{-\frac{N}{2 q_{2}}},\right. \\
\| \nabla\left(a_{i j}(t), J(t), \ell_{i j}(t), A_{i j}(t), J(\mathrm{t}), L_{i j}(t)\left\|_{L_{q}(\Omega)} \leq C \int_{0}^{T} \nabla^{2}(\kappa \mathbf{v}(\cdot, s))\right\|_{L_{q}} \leq C[\mathrm{v}]_{T},\right. \\
\| \partial_{\mathrm{t}}\left(a_{i j}(t), J(t), \ell_{i j}(t), A_{i j}(t), J(\mathrm{t}), L_{i j}(t) \|_{L_{\infty}(\Omega)}\right. \\
\leq C\|\nabla(\kappa \mathbf{v}(\cdot, \mathrm{t}))\|, L_{\infty}(\Omega) \leq C\left(I+[\mathrm{v}]_{T}\right)<t>-\frac{N}{2 q_{2}} \leq C\left(I \quad+[\mathrm{v}]_{T}\right)<t>^{\frac{N}{2 q_{2}}}
\end{gathered}
$$

for any $t \in(0, T]$, where $q \in\left[1, q_{2}\right]$. Moreover, we have

By (36) and (37),

$$
\left(\tilde{a}_{i j}, \tilde{J}, \tilde{\ell}_{i j}, \tilde{A}_{i j}, J, L_{i j}\right)(x, t)=0 \text { for } x \notin B_{2 R} \text { and } t \in[0, T]
$$

$$
\left\|\left(a_{s m}(T) L_{i s}(t) \partial_{t} v_{i}\right)\right\|_{L_{q}(\Omega)} C[v]_{T}+<t>^{-b+\frac{1}{p^{\prime}}}\left\|\partial_{t} v_{i}(t)\right\|_{L_{q_{2}}(\Omega)}
$$

for any $q \in\left[1, q_{2}\right]$. Since $\frac{1}{p^{\prime}}<b-\frac{N}{2 q_{2}}$ as follows from (11), we have

for any $q \in\left[1, q_{2}\right]$

$$
\left.\|<t>^{b} a_{s m}(T) L_{i s}(t) \partial_{t} v_{i}\right) \|_{L_{p}\left((0, T), L_{q}(\Omega)\right)} \leq C\left(I+[v]_{T}^{2}\right)
$$

Next, by Holder's inequality,

$$
<t>^{b}\|\mathbf{v} \cdot \nabla \mathbf{v}(\cdot, t)\|_{L_{q_{1}} / 2}(\Omega) \leq<t>^{\frac{N}{2 q_{1}}}\|\nabla \mathbf{v}(\cdot, t)\|_{L_{q_{1}}(\Omega)}
$$


so that by (36), we have

Since

$$
\left\|<t>^{b} a_{s m}(T) \ell_{i s} a_{k j} v_{j} \frac{\partial v_{i}}{\partial \xi_{k}}\right\|_{L_{p}\left((0, T), L_{q_{1}} / 2(\Omega)\right)} \leq C[\mathbf{v}]_{T}^{2} .
$$

by (36)

Since

$$
\left\langle t>^{b}\|\mathbf{v} \cdot \nabla \mathbf{v}(\cdot, t)\|_{L_{q_{2}}(\Omega)} \leq<t>^{\frac{N}{2 q_{2}}}\|\nabla \mathbf{v}(\cdot, t)\|_{L_{\infty}(\Omega)}<t>^{b-\frac{N}{2 q_{2}}}\|\nabla \mathbf{v}(\cdot, t)\|_{L_{q_{2}}(\Omega)},\right.
$$

$$
\left\|<t>^{b} a_{s m}(T) \ell_{i s} a_{k j} v_{j} \frac{\partial v_{i}}{\partial \xi_{k}}\right\|_{L_{p}\left((0, T), L_{q_{2}}(\Omega)\right)} \leq C\left(I+[\mathbf{v}]_{\mathrm{T}}\right)[\mathbf{v}] \mathrm{T} \leq C\left(I+[\mathbf{v}]_{T}^{2}\right)
$$

by (36)

$$
\frac{\partial}{\partial \xi_{k}}\left(D_{i j}(t) \nabla \mathbf{v}\right)=\sum_{m=1}^{N}\left(A_{m j}(t) \frac{\partial^{2} v_{i}}{\partial \xi_{k} \partial \xi_{m}}+A_{m i}(t) \frac{\partial^{2} v_{j}}{\partial \xi_{k} \partial \xi_{m}}\right)+\sum_{m=1}^{N}\left(\left(\frac{\partial}{\partial \xi_{m}} A_{m j}(t) \frac{\partial v_{i}}{\partial \xi_{m}}+\frac{\partial}{\partial \xi_{k}} A_{m i}(t)\right) \frac{\partial v_{j}}{\partial \xi_{m}}\right)
$$

$$
<t>^{b}\left\|\frac{\partial}{\partial \xi_{k}}\left(D_{i j}(.) \nabla \mathbf{v}\right)\right\|_{L_{q}(\Omega)} \leq C[\mathbf{v}]_{\mathrm{T}}\left\{<t>^{b-\frac{N}{2 q_{2}}}\left\|\nabla^{2} \mathbf{v}(. \mathrm{t})\right\|_{L_{q}(\Omega)}+<t>^{b}\|\nabla \mathbf{v}(\cdot, t)\|_{L_{\infty}(\Omega)}\right\}
$$

for any $q \in\left[1, q_{2}\right]$ and therefore

$$
\left\|<t>^{b} a_{s m}(T) \ell_{i s}(T) a_{k j}(T) \frac{\partial}{\partial \xi_{k}}\left(D_{i j}(.) \nabla \mathbf{v}\right)\right\|_{L_{p}\left((0, T), L_{q}(\Omega)\right)} \leq C[\mathbf{v}]_{T}^{2}
$$

for any $q \in\left[1, q_{2}\right]$. Since

by (36)

$$
\frac{\partial}{\partial \xi_{k}}\left(D_{i j, T}(\mathbf{v})\right)=\sum_{m=1}^{N}\left(a_{m j}(T) \frac{\partial^{2} v_{i}}{\partial \xi_{k} \partial \xi_{m}}+a_{m i}(T) \frac{\partial^{2} v_{j}}{\partial \xi_{k} \partial \xi_{m}}\right)+\sum_{m=1}^{N}\left(\left(\frac{\partial}{\partial \xi_{m}} a_{m j}(T) \frac{\partial v_{i}}{\partial \xi_{m}}+\frac{\partial}{\partial \xi_{k}} a_{m i}(T)\right) \frac{\partial v_{j}}{\partial \xi_{m}}\right),
$$

so that

$$
<t>^{b} \| a_{s m}(T) \ell_{i s}(T) \mathcal{A}_{k j}(t) \frac{\partial}{\partial \xi_{k}}\left(D_{i j, T}(\mathbf{v}) \|_{L_{q}(\Omega)} \leq C[\mathbf{v}]_{\mathrm{T}}\left\{<t>^{b-\frac{N}{2 q_{2}}}\left\|\nabla^{2} \mathbf{v}(. \mathrm{t})\right\|_{L_{q}(\Omega)}+<t>^{b}\|\nabla \mathbf{v}(\cdot, t)\|_{L_{\infty}(\Omega)}\right\},\right.
$$

$$
<t>^{b} \| a_{s m}(T) \ell_{i s}(T) \mathcal{A}_{k j}(T) \frac{\partial}{\partial \xi_{k}}\left(D_{i j, T}(\mathbf{v}) \|_{L_{p}\left((0, T), L_{q}(\Omega)\right)} \leq C[\mathbf{v}]_{T}^{2}\right.
$$

for any $q \in\left[1, q_{2}\right]$. Analogously, we have

$$
\|<t>{ }^{b} a_{s m}(T) L_{i s} a_{k j} \frac{\partial}{\partial \xi_{k}}\left(D_{i j, T}(\mathbf{v}) \|_{L_{p}\left((0, T), L_{q}(\Omega)\right)} \leq C[\mathbf{v}]_{T}^{2}\right.
$$

for any $q \in\left[1, q_{2}\right]$. Summing up, we have obtained (27).

Next, we consider $\mathrm{g}$ and $\mathrm{g}$. To estimate the $H_{p}^{\frac{1}{2}}$ norm, we use the following lemma.

\section{Lemma 5}

Let $f \in H_{\infty}^{1}\left(\mathbb{R}, L_{\infty}(\Omega)\right)$ and $g \in H_{p}^{\frac{1}{2}}\left(\mathbb{R}, L_{q}(\Omega)\right)$. Assume that $f(x, t)=0$ for $(x, t) \notin B_{R} \times \mathbb{R}$.

Then,

$$
\|f g\|_{H_{p}^{2}}^{\frac{1}{2}}\left(\mathbb{R}, L_{q}(\Omega)\right) \leq C\|f\|_{H_{\infty}^{1}\left(\mathbb{R}, L_{\infty}(\Omega)\right)}\|g\|_{H_{p}^{2}}^{\frac{1}{2}}\left(\mathbb{R}, L_{q_{2}}(\Omega)\right)
$$

Proof: To prove the lemma, we use the fact that

$$
H_{p}^{\frac{1}{2}}\left(\mathbb{R}, L_{q}(\Omega)\right)=\left(L_{p}\left(\mathbb{R}, L_{q}(\Omega)\right), H_{p}^{1}\left(\mathbb{R}, L_{q}(\Omega)\right)\right)_{\left[\frac{1}{2}\right]},
$$

where $(\cdot,)_{[\sigma]}$ denotes a complex interpolation functor. Let $q \in\left[1, q_{2}\right]$. Noting that $f(x, t)=0$ for $(x, t) \notin B_{R} \times \mathbb{R}$, we have

$$
\left\|\partial_{t}(f g)\right\|_{L_{q}(\Omega)} \leq\left\|\partial_{t} f\right\|_{L_{\infty}(\Omega)}\|g\|_{L_{q_{2}}(\Omega)}+\|f\|_{L_{\infty}(\Omega)}+\left\|\partial_{t} g\right\|_{L_{q_{2}}(\Omega)},
$$


and therefore

$$
\left\|\partial_{t}(f g)\right\|_{L_{p}\left(\mathbb{R}, L_{q}(\Omega)\right)} \leq C\|f\|_{H_{\infty}^{1}\left(\mathbb{R}, L_{\infty}(\Omega)\right)}\|g\|_{H_{p}^{1}\left(\mathbb{R}, L_{q_{2}}(\Omega)\right)} .
$$

for any $q \in\left[1, q_{2}\right]$. Moreover, we easily see that

$$
\|(f g)\|_{L_{p}\left(\mathbb{R}, L_{q}(\Omega)\right)} \leq C\|f\|_{L_{\infty}\left(\mathbb{R}, L_{\infty}(\Omega)\right)}\|g\|_{L_{p}\left(\mathbb{R}, L_{q_{2}}(\Omega)\right)} .
$$

Thus, by (40), we have (39), which completes the proof of Lemma 5.

To use the maximal $\mathrm{L}_{\mathrm{p}}-\mathrm{L}_{\mathrm{q}}$ estimate, we have to extend $\mathrm{g}$, $\mathrm{g}$ and $\mathrm{h}$ to $\mathrm{R}$. For this purpose, we introduce an extension operator $\tilde{e}_{T}$. Let $f$ be a function defined on $(0, \mathrm{~T})$ such that $\left.f\right|_{t=T}=0$, and then $\tilde{e}_{T}$ is an operator acting on $\mathrm{f}$ defined by

$$
\left[\tilde{e}_{T} f\right](t)= \begin{cases}0 & (t>T), \\ f(t) & (0<t<T), \\ f(-t) & (-T<t<0) \\ 0 & (t<-T)\end{cases}
$$

\section{Lemma 6}

Let $1<p<\infty, 1 \leq q \leq q_{2}$ and $0 \leq a \leq b$. Let $f \in H_{\infty}^{1}\left(0, T, L_{\infty}(\Omega)\right)$ and $g \in H_{p}^{1}\left((0, \mathrm{~T}), L_{q_{2}}(\Omega)\right) \cap L_{p}\left((0, \mathrm{~T}) H_{q}^{2}(\Omega)\right)$. Assume $\left.f\right|_{t=T}=0$ and $f=0$ for $(x, t) \notin B_{R} \times \mathbb{R}$. Let $<t>=\left(1+t^{2}\right)^{1 / 2}$. Then we have

$$
\begin{aligned}
& \left\|\tilde{e}_{T}\left(<t>^{a} f \nabla g\right)\right\|_{H_{p}^{\frac{1}{2}}\left(\mathbb{R}, L_{q}(\Omega)\right)} \leq C<t>^{\frac{N}{2 q_{2}}} f \|_{H_{\infty}^{1}\left((0, \infty), L_{\infty}(\Omega)\right)}
\end{aligned}
$$

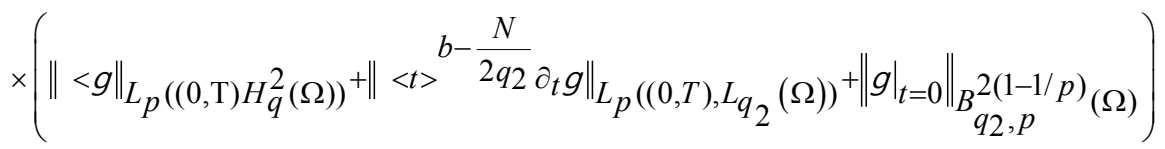

Proof: Let $f_{0}(t)=\left\langle t>^{a-b+\frac{N}{2 q_{2}}} f(t)\right.$ and $g_{0}(t)=<t>^{b+\frac{N}{2 q_{2}}} g(t)$, and then $\left\langle t>^{a} f \nabla g=f_{0} \nabla g_{0}\right.$. Let $T(t)$ be the operator given in (30) and let $h$ be a function in $B_{q_{2}, p}^{2(1-1 / p)}\left(\mathbb{R}^{\mathrm{N}}\right)$ such that $h=\left.g\right|_{t=0}$ in $\Omega$ and $h=\left.g\right|_{t=0}\|h\|_{B_{q_{2}, p}^{2(1-1 / p)}(\Omega)} \leq\left\|\left.g\right|_{t=0}\right\|_{B_{q_{2}, p}^{2(1-1 / p)}(\Omega)}$. Recall the operator $\mathrm{e}_{\mathrm{T}}$ defined in (32) and note that $\left.g_{0}\right|_{t=0}=\left.g\right|_{t=0}=\left.T(\mathrm{t}) h\right|_{t=0}$ in $\Omega$. Let $\tilde{g}(t)=e_{T}\left[g_{0}-T(\cdot) h\right](t)+T(t) h$ for $\mathrm{t}>0$ and let

$$
[l g](t)=\left\{\begin{array}{ll}
\tilde{g}(t) & (t>0), \\
\tilde{g}(-t) & (t<0),
\end{array} \quad[\imath f](t)= \begin{cases}0 & (t>T), \\
f_{0}(t) & (0<t<T), \\
f_{0}(-t) & (-T<t<0), \\
0 & (t<-T)\end{cases}\right.
$$

Since $\tilde{g}(t)=g_{0}(t)$ for $0<t<T$, we have

$$
\tilde{e}_{T}\left[<t>^{a} f \nabla g\right](t)=\left\{\begin{array}{ll}
0 & (t>T), \\
f_{0}(t) \nabla g_{0}(t) & (0<t<T), \\
f_{0}(-t) \nabla g_{0}(-t) & (-T<t<0), \\
0 & (t<-T) .
\end{array}= \begin{cases}0 & (t>T), \\
f_{0}(t) \nabla \tilde{g}(t) & (0<t<T), \\
f_{0}(-t) \nabla \tilde{g}(-t) & (-T<t<0), \\
0 & (t<-T) .\end{cases}\right.
$$

By Lemma 5,

$$
=[l f](t) \nabla[\lg ](t) \text {. }
$$

$$
\left.\left\|\tilde{e}_{T}\left[<t>^{a} f \nabla g\right]\right\|_{H_{p}^{\frac{1}{2}}\left(\mathbb{R}, L_{q}(\Omega)\right)}=\|[\imath f] \nabla[\imath g]\|_{H_{p}^{\frac{1}{2}}\left(\mathbb{R}, L_{q}(\Omega)\right)} \leq C\|[\imath f]\|_{H_{\infty}^{1}\left(\mathbb{R}, L_{q}(\Omega)\right)}\|\nabla(\imath g)\|_{H_{p}^{2}} \frac{1}{R}, L_{q}(\Omega)\right)
$$

Since, $\left.f_{0}\right|_{t=T}=0$ we have

$$
\|[\imath f]\|_{H_{\infty}^{1}\left(\mathbb{R}, L_{q}(\Omega)\right)}=2\left\|\left[f_{0}\right]\right\|_{H_{\infty}\left((0, T), L_{\infty}(\Omega)\right)} \leq\left\|<t>^{\frac{N}{2 q_{2}}} f\right\|_{H_{\infty}^{1}\left((0, T), L_{\infty}(\Omega)\right)},
$$


Because $a-b \leq 0$.

To estimate $\|\nabla(\imath g)\|_{H_{p}^{2}}^{\frac{1}{2}}\left(\mathbb{R}, L_{q}(\Omega)\right)$, we use the fact that $H_{p}^{1}\left(\mathbb{R}, L_{q_{2}}(\Omega)\right) \cap L_{p}\left(\mathbb{R}, H_{q_{2}}^{2}(\Omega)\right)$ is continuously imbedded into $H_{p}^{2}\left(\mathbb{R}, H_{q_{2}}^{1}(\Omega)\right)$ which was proved by Meyries et al., ${ }^{52}$ in case of $\mathrm{p}=\mathrm{q}_{2}$ and by Shibata ${ }^{30}$ for any $1<p, q_{2}<\infty$. Using this fact and (31), we have

$$
\begin{gathered}
\|\nabla(\imath g)\|_{H_{p}^{2}\left(\mathbb{R}, L_{q_{2}}(\Omega)\right)} \leq C\|(\imath g)\|_{H_{p}^{1}}\left(\mathbb{R}, L_{q_{2}}(\Omega)\right)+\|(\imath g)\|_{L_{p}\left(\mathbb{R}, H_{q_{2}}^{2}(\Omega)\right)} \\
\leq C\left(\|\tilde{g}\|_{H_{p}^{1}\left((0, \infty), L_{q_{2}}(\Omega)\right)}=\|\tilde{g}\|_{L_{p}\left((0, \infty), H_{q_{2}}^{2}(\Omega)\right)}\right) \\
\leq C\left(\left\|g_{0}-\mathcal{T}(\cdot) h\right\|_{H_{p}^{1}\left((0, T), L_{q_{2}}(\Omega)\right)}+\left\|g_{0}-\mathcal{T}(\bullet) h\right\|_{L_{p}\left((0, T), H_{q_{2}}^{2}(\Omega)\right)}\right. \\
\left.+\|\mathcal{T}(\cdot) h\|_{H_{p}^{1}\left((0, T), L_{q_{2}}(\Omega)\right)}+\|\mathcal{T}(\bullet) h\|_{L_{p}\left((0, \infty), H_{q_{2}}^{2}(\Omega)\right)}\right) \\
\leq C\left(\left\|<t>{ }^{b-\frac{N}{2 q_{2}}} \partial_{t} g\right\|_{L_{p}\left((0, T), L_{q_{2}}(\Omega)\right)}\right)+\left\|<t>{ }^{b-\frac{N}{2 q_{2}}} \partial_{t} g\right\|_{L_{p}\left((0, T) H_{q}^{2}(\Omega)\right)}+\left\|\left.g\right|_{t=0}\right\|_{B_{q_{2}, p}^{2(1-1 / p)}(\Omega)}
\end{gathered}
$$

This completes the proof of Lemma 6.

Recall the definitions of $\mathrm{g}(\mathrm{v})$ and $\mathrm{h}_{\mathrm{m}}(\mathrm{v})$ given in (24) and (25). By Lemma 6 and (36)

$$
\begin{aligned}
& \| \tilde{e}_{T}\left(<t>^{a} g(\mathrm{v}) \|_{H_{p}^{\frac{1}{2}}\left(\mathbb{R}, L_{q}(\Omega)\right)}\right. \\
& \leq \sum_{j, k=1}^{N}\left\|<t>^{\frac{N}{2 q_{2}}}\left(J(T) A_{k j}(\cdot)+T(\cdot) a_{k j}(\cdot)\right)\right\| H_{\infty}^{1}\left((0, T), L_{\infty}(\Omega)\right) \\
& \times\left(\left\|<t>^{b-\frac{N}{2 q_{2}}} \mathrm{v}\right\|_{L_{p}\left((0, T) H_{q_{2}}^{2}(\Omega)\right)}+\left\|<t>^{b-\frac{N}{2 q_{2}}} \partial_{t} \mathrm{v}\right\|_{L_{p}\left((0, T), L_{q_{2}}(\Omega)\right)}+\left\|\mathrm{u}_{0}\right\|_{B_{q_{2}, p}^{2(1-1 / p)}(\Omega)}\right. \\
& \leq C\left(I+[\mathbf{v}]_{T}^{2}\right)
\end{aligned}
$$

for any $a \in[0, b]$ and $q \in\left[1, q_{2}\right]$. Analogously, we have

$$
\| \tilde{e}_{T}\left(<t>^{a} h(\mathrm{v}) \|_{H_{p}^{\frac{1}{2}}\left(\mathbb{R}, L_{q}(\Omega)\right)} \leq C\left(I+[\mathbf{v}]_{T}^{2}\right)\right.
$$

for any $a \in[0, b]$ and $q \in\left[1, q_{2}\right]$. Analogously, we have

Next, by (36), (37) and (41),

$$
\begin{aligned}
& \left\|\tilde{e}_{T}\left[<t>^{a} g(\mathrm{v})\right]\right\|_{L_{p}}\left(\mathbb{R}, H_{q}^{1}(\Omega)\right) \\
& \leq \sum_{j, k=1}^{N}\left\|<t>^{\frac{N}{2 q_{2}}}\left(J(T) A_{k j}(\cdot)+J(\cdot) a_{k j}(\cdot)\right)\right\|_{L_{\infty}}\left((0, T), L_{\infty}(\Omega)\right)\left\|<t>^{b-\frac{N}{2 q_{2}}} \mathrm{v}\right\|_{L_{p}\left((0, T) H_{q_{2}}^{2}(\Omega)\right)} \\
& \leq C[\mathbf{v}]_{T}^{2}
\end{aligned}
$$

for any $a \in[0, b]$ and $q \in\left[1, q_{2}\right]$. Analogously, we have

$$
\| \tilde{e}_{T}\left(<t>^{b} \mathrm{~h}(\mathrm{v}) \|_{L_{p}\left((0, T) H_{q}^{1}(\Omega)\right)} \leq C[\mathbf{v}]_{T}^{2}\right.
$$

for any $a \in[0, b]$ and $q \in\left[1, q_{2}\right]$. Since

$$
\partial_{t} g_{k}(\mathrm{v})=\sum_{j=1}^{N} \|\left(J(T) \partial_{t} A_{k j}(t)+\left(\partial_{t} J(t) a_{k j}(t)+J(t) \partial_{t} a_{k j}(t)\right) v_{j}+\sum_{j=1}^{N} \|\left(J(T) A_{k j}(t)+J(t) a_{k j}(t)\right) v_{j}\right.
$$


and since $\left\|J(T) a_{k j}(t) J(t)\right\|_{L_{\infty}(\Omega)} \leq C$ as follows from (36), by (37) we have

$$
\begin{aligned}
& \left\|\tilde{e}_{T}\left[<t>^{a} \partial_{t} g_{k}(\mathrm{v})\right]\right\|_{L_{p}}\left((0, T), L_{q}(\Omega)\right) \\
& \leq \sum_{j=1}^{N}\left(<t>^{\frac{N}{2 q_{2}}} \partial_{t}\left(A_{k j}, J, a_{k j}\right)\left\|_{L_{\infty}}\left((0, T), L_{\infty}(\Omega)\right)\right\|<t>^{b-\frac{N}{2 q_{2}}} \mathrm{v} \|_{L_{p}\left((0, T) L_{q_{2}}(\Omega)\right.}\right. \\
& +\left(<t>^{\frac{N}{2 q_{2}}}\left(A_{k j}, J\right)\left\|_{L_{\infty}\left((0, T), L_{\infty}(\Omega)\right)}\right\|<t>^{b-\frac{N}{2 q_{2}}} \partial_{t} \mathrm{v} \|_{L_{p}\left((0, T) L_{q_{2}}(\Omega)\right.},\right.
\end{aligned}
$$

which, combined with (36), furnishes that

$$
\left\|\tilde{e}_{T}\left[<t>^{a} \mathbf{g}(\mathrm{v})\right]\right\|_{L_{p}\left(\mathbb{R}, L_{q}(\Omega)\right)} \leq C\left(I+\underset{(47)}{+[\mathbf{v}]_{T}^{2}}\right)
$$$$
\text { for any } a \in[0, b] \text { and } q \in\left[1, q_{2}\right] \text {. }
$$

\section{Prolongation of local in time solutions}

Before proving Theorem 1, we state a unique existence theorem of locally in time solutions to Eq. (5), which can be proved by a standard argumentation based on maximal $\mathrm{L}_{\mathrm{p}}-\mathrm{L}_{\mathrm{q}}$ regularity theorem for the Stokes equations with free boundary condition..$^{29,40}$

\section{Theorem 7}

Let $N<q_{2}<\infty$ and $2<p<\infty$. Assume that $2 / \mathrm{p}+\mathrm{N} / \mathrm{q}_{2}<1$. Then, given $\mathrm{T}>0$, there exists an $\epsilon_{0}>0$ depending on $\mathrm{T}$ such that if initial data $\mathrm{u}_{0} \in B_{q_{2}, p}^{2(1-1 / p)}(\Omega)$ satisfies the condition:

$$
\begin{gathered}
\left\|\mathrm{u}_{0}\right\|_{B_{q_{2}, p}^{2(1-1 / p)}(\Omega)} \leq \epsilon_{0} \\
\mathbf{v}_{1} \in H_{p}^{1}\left((T, T+1), L_{q_{2}}(\Omega)^{\mathrm{N}}\right) \cap L_{p}\left((T, T+1) H_{q_{2}}^{2}(\Omega)^{\mathrm{N}}\right), q_{1} \in L_{p}\left((T, T+1), H_{q_{2}}^{1}(\Omega) \hat{H}_{q_{2}, 0}^{1}(\Omega)\right)
\end{gathered}
$$

and the compatibility condition (9), then Eq. (5) admits unique solutions $\mathrm{v}$ and $\mathrm{q}$ with

$\mathbf{v} \in H_{p}^{1}\left((0, T), L_{q_{2}}(\Omega)^{\mathrm{N}}\right) \cap L_{p}\left((0, T), H_{q_{2}}^{2}(\Omega)^{\mathrm{N}}\right), q \in L_{p}\left((0, T), H_{q_{2}}^{1}(\Omega)+\hat{H}_{q_{2}, 0}^{1}\right)$

Let $\mathrm{T}$ be a positive number $>2$ and let $\mathrm{v}$ and $\mathrm{q}$ be solutions of Eq. (5) satisfying (13) and (3). In that

view of Theorem 7 , such solutions $\mathrm{v}$ and $\mathrm{q}$ exist uniquely provided

$$
\left\|\mathrm{u}_{0}\right\|_{B_{q_{2}, p}^{2(1-1 / p)}(\Omega)} \leq \epsilon_{0}
$$

Thus, we assume that $0<\epsilon \leq \epsilon_{0}$ in Theorem 1. Let $[\mathrm{v}]_{\mathrm{T}}$ and I be the quantities defined in Theorem 1

in Sect. 1 Introduction. And then, if we prove that there exists a constant $\mathrm{M}>0$ independent of $\epsilon$ and

T such that

$$
[\mathbf{v}]_{T} \leq M\left(I+[\mathbf{v}]_{T}^{2}\right)
$$

then we can prolong $\mathbf{v}$ and q beyond $T$. Namely, there exist $\mathbf{v}_{1}$ and

such that $\mathrm{v}_{1}$ and $\mathrm{q}_{1}$ are solutions to the equations:

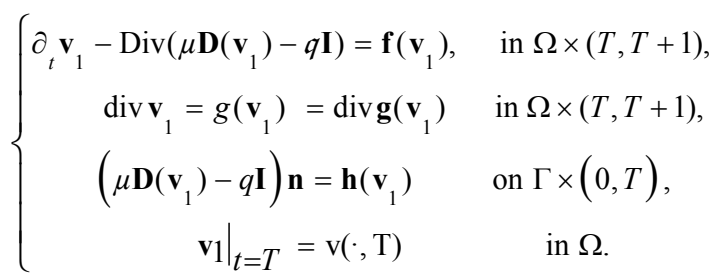

Here, $f\left(\mathrm{v}_{1}\right)$ is consisting of some linear combinations of nonlinear terms of the forms

$$
\begin{aligned}
& V_{0}\left(\int_{0}^{T}\left(\nabla(\kappa \mathbf{v}) d s+\int_{T}^{t}\left(\nabla\left(\kappa \mathbf{v}_{1}\right) d s\right)\right) \nabla^{2} \mathbf{v}_{1}, \quad V_{1}\left(\int_{0}^{T}\left(\nabla(\kappa \mathbf{v}) d s+\int_{T}^{t}\left(\nabla\left(\kappa \mathbf{v}_{1}\right) d s\right) \nabla^{2} \mathbf{v}_{1}\right)\right) \partial_{t} \mathbf{v}_{1}\right. \\
& V_{1}\left(\int_{0}^{T}\left(\nabla(\kappa \mathbf{v}) d s+\int_{T}^{t}\left(\nabla\left(\kappa \mathbf{v}_{1}\right) d s\right)\right)\left(\int_{0}^{T}\left(\nabla(\kappa \mathbf{v}) d s+\int_{T}^{t}\left(\nabla\left(\kappa \mathbf{v}_{1}\right) d s\right) \nabla^{2} \mathbf{v}_{1}\right)\right) \nabla \mathbf{v}_{1},\right. \\
& (1-\kappa)\left({ }^{\bullet} \mathbf{A}\right)-1\left\{\mathbf{v}_{1}\left(\cdot \mathbf{A} \nabla \mathbf{v}_{1}\right)\right\} ;
\end{aligned}
$$

and $g\left(\mathrm{v}_{1}\right), \mathrm{g}\left(\mathrm{v}_{1}\right)$ and $\mathrm{h}\left(\mathrm{v}_{1}\right)$ have the following forms:

$$
\begin{aligned}
& g\left(\mathrm{v}_{1}\right)=V_{3}\left(\int_{0}^{T}\left(\nabla(\kappa \mathbf{v}) d s+\int_{T}^{t}\left(\nabla\left(\kappa \mathbf{v}_{1}\right) d s\right)\right) \nabla \mathbf{v}_{1} ; g\left(\mathrm{v}_{1}\right)=V_{4}\left(\int_{0}^{T}\left(\nabla(\kappa \mathbf{v}) d s+\int_{T}^{t}\left(\nabla\left(\kappa \mathbf{v}_{1}\right) d s\right)\right) \mathbf{v}_{1} ;\right.\right. \\
& \mathrm{h}\left(\mathrm{v}_{1}\right)=V_{s}\left(\int_{0}^{T}\left(\nabla(\kappa \mathbf{v}) d s+\int_{T}^{t}\left(\nabla\left(\kappa \mathbf{v}_{1}\right) d s\right)\right) \nabla \mathbf{v}_{1},\right.
\end{aligned}
$$


where $V_{i}$ are the same nonlinear functions as in (6) and (7).

In fact, the inequality (50) yields that there exists a small constant $\varepsilon \in\left(0, \varepsilon_{0}\right]$ such that if $\mathcal{I} \leq \varepsilon$, then

$$
[\mathbf{v}]_{T} \leq(2 M)^{-1}-\sqrt{(2 M)^{-2}-I}=2 M I+O\left(I^{2}\right) .
$$

Thus, we may assume that

$$
[\mathbf{v}]_{T} \leq 3 M I
$$

By (29) and (52) we have

$$
\|\mathbf{v}(\cdot, T)\| \|_{B_{q_{2}, p}^{2(1-1 / p)}(\Omega)} \leq M_{1} \varepsilon
$$
that

with some positive constant $M_{1}$ independent of $T$. Thus, noting

$$
\int_{0}^{T}\|\nabla \mathbf{v}(\cdot, t)\| L_{\infty}(\Omega) d t \leq C\left(\int_{0}^{T}<t>^{-b p^{\prime}} d s\right)^{1 / p^{\prime}}[\mathbf{v}]_{T} \leq M_{2} \dot{o}
$$

with some positive constant $M_{2}$ independent of $T$, choosing $\varepsilon>0$ smaller if necessary, we can show the

existence of $\mathbf{v}_{1}$ and $\mathrm{q}_{1}$. Thus, setting

$$
\mathrm{v}_{2}(, t)=\left\{\begin{array}{ll}
\mathbf{v}(, t) & 0<t<T, \\
\mathbf{v}_{1}(\cdot, t) & T<t<T+1,
\end{array} q_{2}= \begin{cases}q(, t) & 0<t<T, \\
q_{2}(\cdot, t) & T<t<T+1,\end{cases}\right.
$$

we see that $\mathrm{v}_{2}$ and $\mathrm{q}_{2}$ satisfy the regularity condition:

$\mathrm{v}_{2} \in H_{p}^{1}\left((0, T+1), L_{q_{2}}(\Omega)^{\mathrm{N}}\right) \cap L_{p}\left((0, T+1), H_{q_{2}}^{2}(\Omega)\right), \quad q_{2} \in L_{p}\left((0, T+1), H_{q_{2}}^{1}(\Omega)+\hat{H}_{q_{2,0}}^{1}(\Omega)\right)$

and Eq. (5) replacing $T$ by $T+1$. Repeating this argument, we can prolong $\mathbf{v}$ to time interval $(0, \infty)$.

This completes the proof of Theorem 1. Therefore, we prove (50).

\section{A proof of theorem I}

Let $\mathrm{v}$ and $\mathrm{q}$ be the same $\mathrm{N}$-vector of functions and the function as in Sect. 4. We prove that $\mathrm{v}$ satisfies (50). And, we recall that $\mathrm{T}>2$. As was seen in Sect. 2, v and q satisfy Eq. (26). To estimate v, we write v by, $\mathrm{v}=\mathrm{w}+\mathrm{u}$ where $\mathrm{w}$ is a solution to the equations:

$$
\left\{\begin{array}{rc}
\partial_{t} \mathbf{w}+\lambda_{0} \mathbf{w}-J(T)^{-1} \operatorname{Div} \tilde{\mathbf{S}}(\mathbf{w}, r)=\mathbf{f}(\mathbf{v}) & \text { in } \Omega \times(0, T), \\
\operatorname{div} \mathbf{w}=\tilde{\mathrm{e}}_{\mathrm{T}}[\mathrm{g}(\mathrm{v})]=\operatorname{div} \tilde{\mathrm{e}}_{\mathrm{T}}[\mathrm{g}(\mathrm{v})] & \text { in } \Omega \times(0, T), \\
\tilde{\mathbf{S}}(\mathbf{w}, t)=\tilde{\mathrm{e}}_{\mathrm{T}}[\mathbf{h}] & \text { on } \Gamma \times(0, T), \\
\left.\mathbf{w}\right|_{t=0}=\mathbf{u}_{0} & \text { in } \Omega
\end{array}\right.
$$

with some pressure term $r$, and $\mathbf{u}$ is a solution to the equations:

$$
\left\{\begin{aligned}
\partial_{t} \mathrm{u}-J(T)^{-1} \operatorname{Div} \tilde{\mathbf{S}}(\mathrm{u}, \mathfrak{p})=-\lambda_{0} \mathrm{w} & \text { in } \Omega \times(0, T), \\
\operatorname{div} \mathrm{u}=0 & \text { in } \Omega \times(0, T), \\
\tilde{\mathbf{S}}(\mathrm{u}, \mathfrak{p})=0 & \text { on } \Gamma \times(0, T), \\
\left.\mathrm{u}\right|_{t=0}=0 & \text { in } \Omega
\end{aligned}\right.
$$

To estimate $\mathbf{w}$, we quote the maximal $\mathrm{L}_{\mathrm{p}}-\mathrm{L}_{\mathrm{q}}$ regularity theorem due to Shibata. ${ }^{33}$ Let us consider

$$
\begin{aligned}
& \text { the equations: }
\end{aligned}
$$

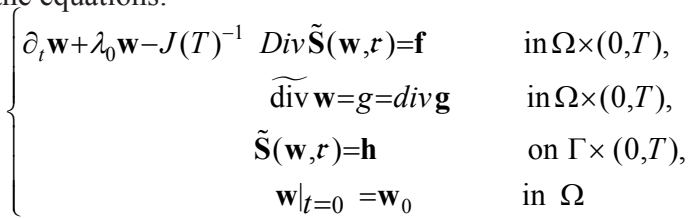

And then, we have

\section{Theorem 8}

Let $\Omega$ be an exterior domain in $\mathbb{R}^{N}$ whose boundary $\Gamma$ is a $\mathrm{C}_{2}$ hyper surface. Let $1<p, q<\infty$ and $\mathrm{T}>0$. Assume that

$\mathrm{w}_{0} \in B_{q, p}^{2(1-1 / p)}(\Omega), \mathrm{f} \in L_{p}\left((0, T), L_{q}(\Omega)^{\mathrm{N}}\right), g \in \stackrel{1}{2}_{p}^{2}\left(\mathbb{R}, L_{q}(\Omega)\right) \cap L_{p}\left(\mathbb{R}, H_{q}^{1}(\Omega)\right)$,

$\mathrm{g} \in H_{p}^{1}\left(\mathbb{R}, L_{q}(\Omega)^{\mathrm{N}}\right), \stackrel{\frac{1}{-}}{\mathrm{h}} \in H_{p}^{2}\left(\mathbb{R}, L_{q}(\Omega)^{\mathrm{N}}\right) \cap L_{p}\left(\mathbb{R}, H_{q}^{1}(\Omega)^{\mathrm{N}}\right)$

and that $\mathrm{w}_{0}$ satisfies the compatibility condition:

$$
\mathrm{w}_{0}-\left.\mathrm{g}\right|_{t=0} \in B_{q, p}^{2(1-1 / p)}(\Omega)
$$

and in addition

$$
\left(\mu \mathbf{D}\left(\mathbf{w}_{0}\right)-\left.\mathbf{h}\right|_{t=0}\right)_{\tau}=0 \text { on } \Gamma
$$

If $2 / p+1 / q<1$, where $d_{\tau}=d-<d, n>n$. Then, there exists a positive number $\lambda_{0}$ such that Eq. (55) admits unique solutions $\mathrm{w}$ and $\mathrm{r}$ with

$\mathbf{w} \in L_{p}\left((0, T), H_{q}^{2}(\Omega)^{\mathrm{N}}\right) \cap H_{p}^{1}\left((0, T), L_{q}(\Omega)^{\mathrm{N}}\right), \mathfrak{r} \in L_{p}\left((0, T), H_{q}^{1}(\Omega)+\hat{H}_{q, 0}^{2}(\Omega)\right)$

Possessing the estimate:

$$
\begin{array}{r}
\|\mathbf{w}\|_{L_{p}\left((0, T), H_{q}^{2}(\Omega)\right)+\left\|\partial_{t} \mathbf{w}\right\|_{L_{p}\left((0, T), L_{q}(\Omega)\right)} \leq C_{q}\left\{\left\|\mathbf{w}_{0}\right\|_{B_{q, p}}(1-1 / p)(\Omega)\right.}+\|\mathbf{f}\| L_{p}\left((0, T), L_{q}(\Omega)\right) \\
\left.+\|(g, \mathbf{h})\|_{H_{p}^{2}}^{\frac{1}{2}}\left(\mathbb{R}, L_{q}(\Omega)\right)\right)\left(\mid(g, \mathbf{h})\left\|L_{p}\left(\mathbb{R}, L_{q}(\Omega)\right)+\right\| \partial_{t} \mathbf{g} \|_{L_{p}}\left(\mathbb{R}, L_{q}(\Omega)\right)\right\},
\end{array}
$$

where $\mathrm{C}$ is a constant that depends on $\mathrm{q}$ but is independent of $\mathrm{T}$. Applying Theorem 8 yields that there exists a large $\lambda_{0}>0$ such that Eq. (53) admits unique solutions $\mathrm{w}$ and $r$ with

$$
\mathbf{w} \in H_{p}^{1}\left((0, T), L_{q}(\Omega)^{\mathrm{N}}\right) \cap L_{p}\left((0, T), H_{q}^{2}(\Omega)^{\mathrm{N}}\right)\left(\mathrm{q} \in \mathrm{q}_{1} / 2, \mathrm{q}_{2}\right)
$$

Possessing the estimate:

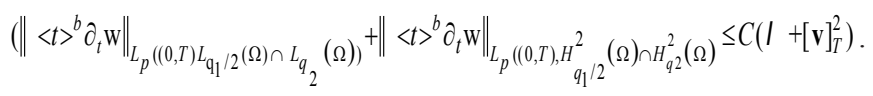

In fact, $\mathbf{f}(\mathbf{v}), \tilde{e}_{T}[g(\mathbf{v})], \tilde{e}_{T}[\mathbf{g}(\mathbf{v})]$ and $\tilde{e}_{T}[\mathbf{h}(\mathbf{v})]$ satisfy (27), (43), (44), (45), (46), and (47), so that we know the existence of $\mathbf{w}$ possessing the estimate:

$\partial_{t} \mathrm{w}\left\|_{L_{p}\left((0, T) L_{q_{1} / 2}(\Omega) \cap L_{q_{2}}(\Omega)\right)}+\right\|\left\langle t>^{b} \partial_{t} \mathrm{w} \|_{L_{p}\left((0, T), H_{q_{1} / 2}^{2}(\Omega) \cap H_{q_{2}}^{2}(\Omega)\right.} \leq C\left(I+[\mathbf{v}]_{T}^{2}\right)\right.$

with some constant $C$ depending on $\mathrm{q}_{1} / 2$ and $\mathrm{q}_{2}$. Let $\mathrm{a}=\min (1, \mathrm{~b})$, and then $\tilde{\mathrm{w}}:=<t>^{a} \mathrm{~W}$ satisfies

the equations:

$\left\{\begin{aligned} \partial_{t} \tilde{\mathbf{w}}+\lambda_{0} \tilde{\mathbf{w}}-J(T)^{-1} \operatorname{Div} \tilde{\mathbf{S}}\left(\tilde{\mathbf{w}},<t>^{a} t\right)=\left\langle t>^{a} \mathbf{f}+\lambda_{0} a t<t>^{a-2} \mathbf{w}\right. & \text { in } \Omega \times(0, T), \\ \widetilde{\operatorname{div}} \tilde{\mathbf{w}}=\tilde{e}_{T}\left[<t>^{a} g(\mathbf{v})\right]=\operatorname{div} \tilde{e}_{T}\left[<t>^{a} g(\mathbf{v})\right] & \text { in } \Omega \times(0, T), \\ \tilde{\mathbf{S}}\left(\tilde{\mathbf{w}},<t>^{a} t\right)=\tilde{e}_{T}\left[<t>^{a} \mathbf{h}\right] & \text { on } \Gamma \times(0, T), \\ \left.\tilde{\mathbf{w}}\right|_{t=0}=\mathrm{u}_{0} & \text { in } \Omega\end{aligned}\right.$


Since

$$
\left\|t<t>^{a-2} \mathbf{w}\right\|_{L_{p}\left((0, T), L_{q}(\Omega)\right)} \leq\|\mathbf{w}\|_{L_{p}\left((0, T), L_{q}(\Omega)\right)} \leq C\left(I+[\mathbf{v}]_{T}^{2}\right)
$$

as follows from the fact that $a-1 \leq 0$, we have

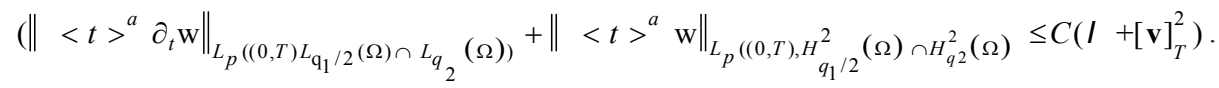

Repeating this argument finite times yields (56). In particular, by (56) we have

$$
[\mathrm{w}]_{\mathrm{T}} \leq C\left(I+[\mathbf{v}]_{T}^{2}\right)
$$

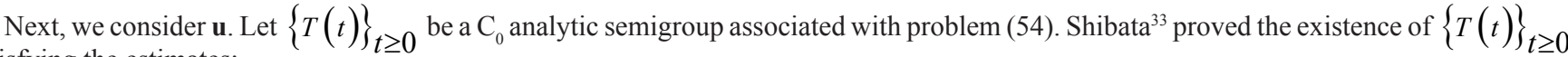
satisfying the estimates:

$$
\|T(t) \mathbf{f}\|_{L_{p}(\Omega)} \leq C t^{-\frac{N}{2}\left(\frac{1}{q}-\frac{1}{p}\right)}\|\mathbf{f}\|_{L_{q}(\Omega)},\|\Delta T(t) \mathbf{f}\|_{L_{p}(\Omega)} \leq C t^{-\frac{1}{2}-\frac{N}{2}\left(\frac{1}{q}-\frac{1}{p}\right)}\|\mathbf{f}\|_{L_{q}(\Omega)}
$$

for any $\mathrm{t}>0$ and $\mathrm{f} \in L_{q}(\Omega)^{N}$ provided that $1<q \leq p \leq \infty$ and $q \leq q_{2}$. To represent $\mathrm{u}$ by using $\{T(t)\}_{t \geq 0}$, we introduce the solenoidal space $J_{q}(\Omega)$ defined by

$$
J_{q}(\Omega)=\left\{\mathrm{f} \in L_{q}(\Omega)^{N} \mid\left(\mathrm{f}, J{ }^{T} \mathbf{A} \nabla \varphi\right)_{\Omega}=0 \text { for any } \varphi \in \hat{H}_{q^{\prime}, 0}^{1}(\Omega)\right\}
$$

Here, $\boldsymbol{A}$ is the matrix defined in (4) and $\mathrm{J}$ the function given in (15), and

$$
\hat{H}_{q^{\prime}, 0}^{1}(\Omega)=\left\{\varphi \in L_{q^{\prime}, l o c}(\Omega)\left|\nabla \varphi L_{q^{\prime}}(\Omega)^{N}, \quad \varphi\right|_{\Gamma}=0\right\} .
$$

As was proved by Shibata ${ }^{30}$ we know that for any $\mathrm{f} \in L_{q}(\Omega)^{N}$ there exists a unique solution $\psi \in \hat{H}_{q^{\prime}, 0}^{1}(\Omega)$ of the variational equation

$$
\left(\mathbf{A} \nabla \psi, J{ }^{T} \mathbf{A} \varphi\right)_{\Omega}=\left(\mathrm{f}, J{ }^{T} \mathbf{A} \nabla \varphi\right)_{\Omega}=0 \text { for any } \varphi \in \hat{H}_{q^{\prime}, 0}^{1}(\Omega) .
$$

which possesses the estimate $\|\nabla \psi\|_{L_{q}}(\Omega) \leq C_{q}\|\mathbf{f}\|_{L_{q}}(\Omega)$ Here $\mathrm{C}_{\mathrm{q}}$ is a constant that is independent of $\mathrm{v}$

and $T$ in view of (36). Given $\mathrm{f} \in L_{q}(\Omega)^{N}$ let $\psi \in \hat{H}_{q^{\prime}, 0}^{1}(\Omega)$ be a unique solution of Eq.(61), and let $\mathbf{P}_{q}$ be an operator acting on $\mathbf{f}$ defined $\mathbf{P}_{q} \mathbf{f}=\mathbf{f}-\nabla \psi$. And then, $\mathbf{P}_{q} \mathbf{f} \in J_{q}(\Omega)$ and

$$
\left\|\mathbf{P}_{q} \mathbf{f}\right\|_{L_{q}(\Omega)} \leq C_{q}\|\mathbf{f}\|_{L_{q}(\Omega)}
$$

with some constant $\mathrm{C}_{\mathrm{g}}$ that is independent of $\mathrm{v}$ and $\mathrm{T}$. By Proposition 21 in Shibaata, ${ }^{33}$ we have

$$
\mathbf{u}(\cdot, t)=-\lambda_{0} \int_{0}^{t} T(t-s)(\mathbf{P w})(\cdot, s) d s .
$$

Combining (59) and (62) yields that

$$
\begin{aligned}
\left\|\nabla^{j} \mathrm{u}(\cdot, t)\right\|_{L_{r}(\Omega)} \leq & C_{r, \tilde{q}_{1}} \int_{0}^{t-1}(t-s)^{-\frac{j}{2}-\frac{N}{2}\left(\frac{1}{\tilde{q}_{1}}-\frac{1}{r}\right)}\|\mathbf{w}(\cdot, s)\|_{L_{\tilde{q}_{1}}}(\Omega) d s \\
& +C_{r, \tilde{q}_{2}} \int_{0}^{t-1}(t-s)^{-\frac{j}{2}-\frac{N}{2}\left(\frac{1}{\tilde{q}_{2}}-\frac{1}{r}\right)}\|\mathbf{w}(\cdot, s)\|_{L_{\tilde{q}_{2}}}(\Omega) d s
\end{aligned}
$$

for $\mathrm{j}=0,1$, for any $\mathrm{t}>1$ and for any indices $r, \tilde{q}_{1}$ and $\tilde{q}_{2}$ such that $1<\tilde{q}_{1}, \tilde{q}_{2} \leq r \leq \infty$ and $\tilde{q}_{1}, \tilde{q}_{2} \leq q_{2}$, where $\nabla^{0} u=u$ and $\nabla^{1} u=\nabla u$.

Recall that $\mathrm{T}>2$. In what follows, we prove that

$$
\begin{gathered}
\left(\int_{2}^{T}\left(<t>^{b}\|\mathbf{u}(\cdot, t)\| H_{\infty}^{1}(\Omega)\right)^{\mathrm{p}} d t\right)^{1 / p} \leq C\left(I+[\mathbf{v}]_{T}^{2}\right) \\
\underset{2 \leq t \leq T}{\operatorname{Sup}\left(<t>^{\frac{N}{2 q_{1}}}\|\mathbf{u}(\cdot, t)\|_{L_{q_{1}}} \leq C\left(I+[\mathbf{v}]_{T}^{2}\right)\right.}
\end{gathered}
$$

$$
\left(\int_{2}^{T}\left(\langle t\rangle^{b-\frac{N}{2 q_{1}}}\|\mathbf{u}(\cdot, t)\|_{q_{1}}^{1}(\Omega)\right)^{\mathrm{p}} d t\right)^{1 / p} \leq C\left(I+[\mathbf{v}]_{T}^{2}\right)
$$

$$
\left(\int_{2}^{T}\left(<t>{ }^{b-\frac{N}{2 q_{2}}}\|\mathrm{u}(\cdot, t)\| H_{q_{2}}^{1}(\Omega)\right)^{\mathrm{p}} d t\right)^{1 / p} \leq C\left(I+[\mathbf{v}]_{T}^{2}\right)
$$

By (64) with $r=\infty, \tilde{q}_{1}=q_{1} / 2$ and $\tilde{q}_{2}=q_{2}$,

$\|\mathbf{u}(\cdot, t)\|_{H_{\infty}^{1}(\Omega)} \leq C \int_{0}^{t}\|T(\mathrm{t}-\mathrm{s}) \mathbf{w}(\cdot, s)\|_{H_{\infty}^{1}(\Omega)} d s=C\left(I_{\infty}(t)+I I_{\infty}(t)+I I I_{\infty}(t)\right)$

With

$$
\begin{aligned}
& I_{\infty}(t)=\int_{0}^{t / 2}(\mathrm{t}-\mathrm{s})^{-\frac{N}{q_{1}}}\|\mathbf{w}(\cdot, s)\|_{L_{q_{1} / 2}(\Omega)} d s, \\
& I I_{\infty}(t)=\int_{t / 2}^{t-1}(\mathrm{t}-\mathrm{s})^{-\frac{N}{q_{1}}}\|\mathbf{w}(\cdot s)\|_{L_{q_{1} / 2}(\Omega)} d s, \\
& I I I_{\infty}(t)=\int_{t-1}^{t}(\mathrm{t}-\mathrm{s})^{-\frac{N}{2 q_{2}}}-\frac{1}{2}\|\mathbf{w}(\cdot s)\|_{L_{q_{2} / 2}(\Omega)} d s .
\end{aligned}
$$

Since 


$$
\begin{aligned}
I_{\infty}(t) & \leq(t / 2)^{-\frac{N}{q_{1}}}\left(\int_{0}^{t / 2}<s>^{-b p^{\prime}} d s\right)^{1 / p^{\prime}}\left(\int_{0}^{t / 2}<s>^{b}\|\mathbf{w}(\cdot, s)\|_{L_{q_{1} / 2}(\Omega)^{p}} d s\right)^{1 / p} \\
& \leq C\left(b p^{\prime}-1\right)^{-1 / p^{\prime}}\left(I+[\mathbf{v}]_{T}^{2}\right) \mathrm{t}^{-\frac{N}{q_{1}}}
\end{aligned}
$$

as follows from the condition: $b p^{\prime}>1$ in (11), by the condition: $\left(\frac{N}{q_{1}}-b\right) p>1$ in (11), we have

$$
\int_{2}^{T}\left(<t>^{b} I_{\infty}(t)\right)^{p} d t \leq C \int_{2}^{T}<t>^{-\left(\frac{N}{q_{1}}-b\right) p} d t\left(I+[\mathbf{v}]_{T}^{2}\right)^{p} \leq C\left(\left(\frac{N}{q_{1}}-b\right) p-1\right)^{-1}\left(I+[\mathbf{v}]_{T}^{2}\right)^{p} .
$$

By Holder's inequality

$$
\begin{aligned}
<t>^{b} I I_{\infty}(t) \leq & C \int_{t / 2}^{t-1}(t-s)^{-\frac{N}{q_{1}}}<s>^{b}\|\mathbf{w}(\cdot, s)\|_{L_{q_{1} / 2}}(\Omega) d s \\
& \leq C\left(\int_{t / 2}^{t-1}(t-s)^{-\frac{N}{q_{1}}} d s\right)^{1 / p^{\prime}}\left(\int_{t / 2}^{t-1}(t-s)^{-\frac{N}{q_{1}}}<s>^{b}\|\mathbf{w}(\cdot, s)\|_{L_{q_{1} / 2}}(\Omega)^{d s)^{1 / p}}\right. \\
& \leq C\left(\frac{N}{q_{1}}-1\right)^{-1 / p^{\prime}}\left(\int_{t / 2}^{t-1}(t-s)^{-\frac{N}{q_{1}}}<s>^{b}\|\mathbf{w}(\cdot, s)\|_{L_{q_{1} / 2}}(\Omega)^{d s)^{1 / p}}\right.
\end{aligned}
$$

Because $N / q_{1}=N / q_{2}+1>1$. By the change of integration order and (56),

$$
\begin{aligned}
\int_{2}^{T}\left(\left\langle t>^{b} I I_{\infty}(t)\right)^{p}\right. & \leq C\left(\frac{N}{q_{1}}-1\right)^{-\frac{p}{p^{\prime}}} \int_{2}^{T} d t \int_{t / 2}^{t-1}(t-s)^{-\frac{N}{q_{1}}}\left(<s>^{b}\|\mathbf{w}(\cdot, s)\|_{L_{q_{1} / 2}(\Omega)}\right)^{p} d s \\
& \leq C\left(\frac{N}{q_{1}}-1\right)^{-\frac{p}{p^{\prime}}} \int_{1}^{T-1}\left(<s>^{b}\|\mathbf{w}(\cdot, s)\|_{L_{q_{1} / 2}(\Omega)}\right)^{\mathrm{p}} d s \int_{s+1}^{2 s}(t-s)^{-\frac{N}{q_{1}}} d t \\
& \leq C\left(\frac{N}{q_{1}}-1\right)^{-p}\left(I+[\mathbf{v}]_{T}^{2}\right)^{p} .
\end{aligned}
$$

Since $\frac{N}{2 q_{2}}+\frac{1}{2}<1$ as follows from $\mathrm{q}_{2}>\mathrm{N}$, by Holder's inequality,

$$
\begin{aligned}
<t>^{b} I I I_{\infty}(t) \leq & C \int_{t-1}^{t}(t-s)^{-\frac{N}{2 q_{2}}-\frac{1}{2}}\left\langle s>^{b}\|\mathbf{w}(\cdot, s)\|_{L_{q_{2}}}(\Omega)^{d s}\right. \\
& \leq C\left(\int_{t-1}^{t}(t-s)^{-\frac{N}{2 q_{2}}-\frac{1}{2}} d s\right)^{1 / p^{\prime}}\left(\int_{t-1}^{t}(t-s)^{-\frac{N}{2 q_{2}}-\frac{1}{2}}(<s\rangle^{b}\|\mathbf{w}(\cdot, s)\|_{L_{q_{2}}}(\Omega)^{p} d s\right)^{1 / p} \\
& \leq C\left(-\frac{N}{2 q 2}-\frac{1}{2}\right)^{-1 / p^{\prime}}\left(\int_{t-1}^{t}(t-s)^{-\frac{N}{2 q_{2}}-\frac{1}{2}}(<s\rangle^{b}\|\mathbf{w}(\cdot, s)\|_{L_{q_{2}}}(\Omega)^{p} d s\right)^{1 / p} .
\end{aligned}
$$

By the change of integration order, we have

$$
\begin{aligned}
\int_{2}^{T}\left(\left\langle t>^{b} I I I_{\infty}(t)\right)^{p} d t\right. & \leq C\left(1-\frac{N}{2 q_{2}}\right)^{-\frac{p}{p^{\prime}}} \int_{2}^{T} d t \int_{t-1}^{t}(t-s)^{-\frac{N}{2 q_{2}}-\frac{1}{2}}\left(<s>^{b}\|\mathbf{w}(\cdot, s)\|_{L_{q_{2}}}(\Omega)\right)^{p} d s \\
& \leq C\left(1-\frac{N}{2 q_{2}}\right)^{-\frac{p}{p^{\prime}}} \int_{1}^{T}\left(<s>^{b}\|\mathbf{w}(\cdot, s)\|_{L_{q_{2}}}(\Omega)^{p} d s \int_{s}^{s+1}(t-s)^{-\frac{N}{2 q_{2}}-\frac{1}{2}} d t\right. \\
& \leq C\left(1-\frac{N}{2 q_{2}}\right)^{-p}\left(I+[\mathbf{v}]_{T}^{2}\right)^{p}
\end{aligned}
$$


Summing up, we have obtained (65). Next, we prove (66).

By (64) with $r=q_{1}, \tilde{q}_{1}=q_{1} / 2$ and $\tilde{q}_{2}=q_{1}$,

With

$$
\|\mathbf{u}(\cdot, \mathrm{t})\|_{L_{q_{1}}(\Omega)} \leq C\left(I_{q_{1}, \infty}(t)+I I_{q_{1}, 1}(t)+I I I_{q_{1}, 1}(t)\right)
$$

$$
\begin{aligned}
& I_{q_{1}, 1}(t)=\int_{0}^{t / 2}(t-s)^{-\frac{N}{2 q_{1}}}\|\mathbf{w}(\cdot s)\|_{L_{q_{1} / 2}(\Omega)} d s, \\
& I I_{q_{1}, 1}(t)=\int_{t / 2}^{t-1}(t-s)^{-\frac{N}{2 q_{1}}}\|\mathbf{w}(\cdot, s)\|_{L_{q_{1} / 2}(\Omega)} d s, \\
& I I I_{q_{1}, 1}(t)=\int_{t-1}^{t}\|\mathbf{w}(\cdot, s)\|_{L_{q_{1}}}(\Omega)^{d s .}
\end{aligned}
$$

By (56)

$$
\begin{aligned}
I_{q_{1}, 1}(t) & \left.\leq(t / 2)^{-\frac{N}{2 q_{1}}}\left(\int_{0}^{t / 2}<s>^{-b p^{\prime}} d s\right)^{1 / p^{\prime}}\left(\int_{0}^{t / 2}<s>^{b}\|\mathbf{w}(\cdot, s)\|_{L_{q_{1} / 2}(\Omega)}\right)^{p} d s\right)^{1 / p} \\
& \leq C t^{-\frac{N}{2 q_{1}}}\left(I+[v]_{T}^{2}\right)^{p} .
\end{aligned}
$$

Analogously, by Holder's inequality and (56),

$$
\begin{aligned}
& I_{q_{1}, 1}(t)=\int_{t / 2}^{t-1}(t-s)^{-\frac{N}{2 q_{1}}}<s>^{-b}<s>^{b}\|\mathbf{w}(\cdot s)\|_{L_{q_{1} / 2}}(\Omega) d s, \\
& \leq C<t>^{-b}\left(\int_{t / 2}^{t-1}(t-s)^{-\frac{N p^{\prime}}{2 q_{1}}} d s\right)^{1 / p^{\prime}}\left(\int_{0}^{T}\left(<s>^{b}\|\mathbf{w}(\cdot, s)\|_{L_{q_{1} / 2}(\Omega)^{2}}\right)^{p} d s\right)^{1 / p} \\
& =C\left(1--\frac{N p^{\prime}}{2 q_{1}}\right)^{1 / p^{\prime}}<t>^{-b-\frac{N}{2 q_{1}}+\frac{1}{p^{\prime}}}\left(I+[\mathbf{v}]_{T}^{2}\right) \\
& \leq C\left(1--\frac{N p^{\prime}}{2 q_{1}}\right)^{1 / p^{\prime}}<t>^{\frac{N}{2 q_{1}}}\left(I+[\mathbf{v}]_{T}^{2}\right) \\
& \text { because } b>\frac{1}{p^{\prime}} . \text { Finally, by }(56),
\end{aligned}
$$

$$
\begin{aligned}
I I I_{q_{1}, 1}(t) & \leq C t^{-b} \int_{t-1}^{t}<s>^{b}\|\mathbf{w}(\cdot s)\|_{L_{q_{1} / 2}}(\Omega) d s \\
& \left.\left.\leq C t^{-b}\left(\int_{t-1}^{t} d s\right)^{1 / p^{\prime}}\right)\left(\int_{0}^{T}<s>^{b}\|\mathbf{w}(\cdot, s)\|_{L_{q_{1} / 2}(\Omega)^{p}}\right)^{p} d s\right)^{1 / p} \\
& \leq C t^{-b}\left(I+[\mathbf{v}]_{T}^{2}\right) .
\end{aligned}
$$

Summing up, we have obtained (66). Next, we prove (67). By (64)

$$
\|\mathbf{u}(\cdot, \mathrm{t})\|_{H_{q_{1}}^{1}(\Omega)} \leq C\left(I_{q_{1}, 2}(t)+I I_{q_{1}, 2}(t)+I I I_{q_{1}, 2}(t)\right)
$$

with

$$
\begin{aligned}
& I_{q_{1}, 2}(t)=\int_{0}^{t / 2}(t-s)^{-\frac{N}{2 q_{1}}}\|\mathbf{w}(\cdot, s)\|_{L_{q_{1} / 2}(\Omega)} d s, \\
& I I_{q_{1}, 2}(t)=\int_{t / 2}^{t-1}(t-s)^{-\frac{N}{2 q_{1}}}\|\mathbf{w}(\cdot, s)\|_{L_{q_{1} / 2}(\Omega)} d s, \\
& I I I_{q_{1}, 2}(t)=\int_{t-1}^{t}(t-s)^{-\frac{1}{2}}\|\mathbf{w}(\cdot, s)\|_{L_{q_{1}}(\Omega)} d s .
\end{aligned}
$$

By (56),

$$
\begin{aligned}
I_{q_{1}, 2}(t) & \leq(t / 2)^{-\frac{N}{2 q_{1}}}\left(\int_{0}^{t / 2}\left\langle s>^{-b p^{\prime}} d s\right)^{1 / p^{\prime}}\left(\int_{0}^{t / 2}\langle s\rangle^{b}\|\mathbf{w}(\cdot, s)\|_{L_{q_{1} / 2}(\Omega)}\right)^{p} d s\right)^{1 / p} \\
& \leq C t^{-\frac{N}{2 q_{1}}}\left(I+[v]_{T}^{2}\right) .
\end{aligned}
$$

so that by the condition: $\left(\frac{N}{q 1}-b\right) p>1$ in (11)

$$
\left(\int_{2}^{T}\left(<t>^{b-\frac{N}{2 q_{1}}} I_{q_{1}, 2}(t)\right)^{p} d t\right)^{1 / p} \leq C\left(\left(\frac{N}{q_{1}}-b\right) p-1\right)^{-1 / p}\left(I+[\mathbf{v}]_{T}^{2}\right) .
$$

By Holder's inequality,

$$
\begin{aligned}
& \langle t\rangle^{b-\frac{N}{2 q_{1}}} I I_{q_{1}, 2}(t) \leq C<t>^{-\frac{N}{2 q_{1}}} \int_{t / 2}^{t-1}(t-s)^{-\frac{N}{2 q_{1}}}<s>^{b}\|\mathbf{w}(\cdot s)\|_{L_{q_{1} / 2}(\Omega)} d s \\
& \leq C<t>^{-\frac{N}{2 q_{1}}}\left(\int_{t / 2}^{t-1}(t-s)^{-\frac{N p^{\prime}}{2 q_{1}}} d s\right)^{1 / p^{\prime}}\left(\int _ { o } ^ { T } \left(\langle s\rangle^{b}\|\mathbf{w}(\cdot, s)\|_{\left.L_{q_{1} / 2}(\Omega)^{p} d s\right)^{1 / p}}\right.\right. \\
& \leq C(1+t)^{-\left(\frac{N}{q_{1}}-\frac{1}{p^{\prime}}\right)}\left(I+[\mathbf{v}]_{T}^{2}\right) .
\end{aligned}
$$

$\begin{aligned} & \text { Since } \\ & \text { have }\end{aligned}\left(\frac{N}{q_{1}}-\frac{1}{p^{\prime}}\right) p>1$ as follows from $\frac{N}{q_{1}}=1+\frac{N}{q_{2}}>1=\frac{1}{p}+\frac{1}{p^{\prime}}$, we

$$
\left(\int_{2}^{T}\left(<t>>^{b-\frac{N}{q_{1}}} I I_{q_{1}, 2}(t)\right)^{p} d t\right)^{1 / p} \leq C\left(\left(\frac{N}{q_{1}}-b\right) p-1\right)^{-1 / p}\left(I+[\mathbf{v}]_{T}^{2}\right)
$$

Since $q_{1} / 2<q_{1}<q_{2}$, we have

$$
\|\mathbf{w}(\cdot, t)\|_{L_{q_{1}}(\Omega)} \leq\|\mathbf{w}(\cdot, t)\|_{L_{q_{1} / 2}^{N+2 q_{2}}(\Omega)}^{\frac{q_{2}}{N+2}} \quad\|\mathbf{w}(\cdot, t)\|_{L_{q_{2}}(\Omega)}^{\frac{N+q_{2}}{N+2 q_{2}}}
$$

Let $\alpha=\frac{q_{2}}{N+2 q_{2}}$ and $\beta=\frac{N+q_{2}}{N+2 q_{2}}$ then $\alpha+\beta=1$, so that by (56) and Holder's inequality

$\left\|<t>^{b} \mathbf{w}\right\|_{L_{p}\left((0, \mathrm{~T}), L_{q_{1}}(\Omega)\right)}$

$\left.\leq\left(\int_{0}^{T}\left(\langle t\rangle^{b}\|\mathbf{w}(\cdot, t)\|_{L_{q_{1} / 2}(\Omega)}\right)^{p \alpha}(<t\rangle^{b}\|\mathbf{w}(\cdot, t)\|_{L_{q_{2}}(\Omega)}\right)^{p \beta} d t\right)^{1 / p}$

$\left.\left.\leq\left(\int_{0}^{T}(<t\rangle^{b}\|\mathbf{w}(\cdot, t)\|_{L_{q_{1} / 2}(\Omega)}\right)^{p} d t\right)^{\alpha / p}\left(\int_{0}^{T}(<t\rangle^{b}\|\mathbf{w}(\cdot, t)\|_{L_{q_{2}}}(\Omega)\right)^{p} d t\right)^{\beta / p}$

$\leq C\left(I+[\mathbf{v}]_{T}^{2}\right)$

$$
\begin{aligned}
& \text { Since } \\
& <t>^{b-\frac{N}{2 q_{1}}} I I I I_{q_{1}, 2}(t) \leq \int_{t-1}^{t}(t-s)^{-\frac{1}{2}}\left\langle s>^{b-\frac{N}{2 q_{1}}}\|\mathbf{w}(\cdot, s)\|_{q_{1}}(\Omega) d s\right. \\
& \quad \leq\left(\int_{t-1}^{t}(t-s)^{-\frac{1}{2}} d s\right)^{1 / p^{\prime}}\left(\int_{t-1}^{t}(t-s)^{-\frac{1}{2}} d s\left(\langle s\rangle^{b}\|\mathbf{w}(\cdot, s)\|_{L_{q_{1}}}(\Omega)^{p} d s\right)^{1 / p},\right.
\end{aligned}
$$


by the change of integration order, we have

$\int_{2}^{T}\left(\langle t\rangle^{b-\frac{N}{2 q_{1}}} I I I I_{q_{1}, 2}(t)\right)^{p} d t \leq 2^{\frac{p}{p^{\prime}}} \int_{2}^{T} d t \int_{t-1}^{t}(t-s)^{-\frac{1}{2}}\left(\langle s\rangle^{b}\|\mathbf{w}(\cdot, s)\|_{q_{q_{1}}}(\Omega)\right)^{p} d s$

$\leq 2^{\frac{p}{p^{\prime}}} \int_{0}^{T}\left(\langle s\rangle^{b}\|\mathbf{w}(\cdot s)\|_{L_{q_{1}}}(\Omega)\right)^{p} d s \int_{s}^{s+1}(t-s)^{-\frac{1}{2}} d t=2^{p}\left\|\langle t\rangle^{b} \mathbf{w}\right\|_{L_{p}\left((0, T), L_{q_{1}}(\Omega)\right)}$,

which, combined with (69), furnishes that

$$
\left(\int_{2}^{T}\left(<t>^{b-\frac{N}{2 q_{1}}} I I I_{q_{1}, 2}(t)\right)^{p} d t\right)^{1 / p} \leq C\left(I+[\mathbf{v}]_{T}^{2}\right)
$$

Summing up, we have obtained (67).

Finally, we prove (68). By (64) with $r=q_{2}, \tilde{q}_{1}=q_{1} / 2$ and $\tilde{q}_{2}=q_{2}$

$$
\|\mathbf{u}(\cdot, t)\|_{L_{q_{2}}(\Omega)} \leq C\left(I_{q_{2}}(t)+I I_{q_{2}}(t)+I I I_{q_{2}}(t)\right)
$$

with

$$
\begin{aligned}
& I_{q_{2}}(t)=\int_{0}^{t / 2}(t-s)^{-\frac{N}{2}\left(\frac{2}{q_{1}}+\frac{1}{q_{2}}\right)}\|\mathbf{w}(\cdot, s)\|_{L_{q_{1} / 2}(\Omega)} d s, \\
& I I_{q_{2}}(t)=\int_{t / 2}^{t-1}(t-s)^{-\frac{N}{2}\left(\frac{2}{q_{1}}+\frac{1}{q_{2}}\right)}\|\mathbf{w}(\cdot, s)\|_{L_{q_{1} / 2}(\Omega)} d s, \\
& I I I_{q_{2}}(t)=\int_{t-1}^{t}\|\mathbf{w}(\cdot, s)\|_{L_{q_{2}}(\Omega)} d s .
\end{aligned}
$$

By Holder's inequality,

$$
\begin{aligned}
I_{q_{2}}(t) & \leq(t / 2)^{-\frac{N}{2}\left(\frac{2}{q_{1}}+\frac{1}{q_{2}}\right)}\left(\int_{0}^{t / 2}\langle s\rangle^{b p^{\prime}} d s\right)^{1 / p^{\prime}}\left(\int_{0}^{t / 2}\langle s\rangle^{b}\|\mathbf{w}(\cdot s)\|_{L_{q_{1} / 2}}(\Omega)^{p} d s\right)^{1 / p} \\
& \leq C<t>^{-\frac{N}{2}\left(\frac{2}{q_{1}}+\frac{1}{q_{2}}\right)}\left(I+[\mathbf{v}]_{T}^{2}\right) .
\end{aligned}
$$

for $t \geq 2$. Since $\frac{N}{2}\left(\frac{2}{q_{1}}+\frac{1}{q_{2}}\right)-\left(b-\frac{N}{2 q_{2}}\right)=\frac{N}{q_{1}}-b$

by the condition: $\left(\frac{N}{q_{1}}-b\right) p>1$ in (11),

$$
\begin{aligned}
\left(\int_{2}^{T}\left(<t>^{b-\frac{N}{2 q_{2}}} I_{q_{2}}(t)\right)^{p} d t\right)^{1 / p} & \leq C\left(\int_{2}^{T} t^{-\left(\frac{N}{q_{1}}-b\right) p} d t\right)^{1 / p}\left(I+[\mathbf{v}]_{T}^{2}\right) \\
& \leq C\left(\left(\frac{N}{q_{1}}-b\right) p-1\right)^{-1 / p}\left(I+[\mathbf{v}]_{T}^{2}\right) .
\end{aligned}
$$

Since

$$
\frac{N}{2}\left(\frac{2}{q_{1}}-\frac{1}{q_{2}}\right)=\frac{N}{2}\left(\frac{1}{q_{2}}+\frac{2}{N}\right)=\frac{N}{2 q_{2}}+1>1,
$$

by Holder's inequality

$$
\begin{aligned}
\langle t\rangle^{b-\frac{N}{2 q_{2}}} I I_{q_{2}}(t) & \leq C \int_{t / 2}^{t-1}(t-s)^{-\left(\frac{N}{2 q_{2}}+1\right)}\langle s\rangle^{b-\frac{N}{2 q_{2}}}\|\mathbf{W}(\cdot s)\|_{L_{q_{1} / 2}}(\Omega) d s \\
& \leq C\left(\int_{t / 2}^{t-1}(t-s)^{-\left(\frac{N}{2 q_{2}}+1\right)} d s\right)^{1 / p^{\prime}}\left(\int_{t / 2}^{t-1}(t-s)^{-\left(\frac{N}{2 q_{2}}+1\right)}\left(\langle s\rangle^{b}\|\mathbf{W}(\cdot s)\|_{L_{q_{1} / 2}(\Omega)}(\Omega) d s\right)^{1 / p}\right. \\
& \leq C\left(\frac{N}{2 q_{2}}\right)^{-1 / p^{\prime}}\left(\int_{t / 2}^{t-1}(t-s)^{-\left(\frac{N}{2 q_{2}}+1\right)}\left(\langle s\rangle^{b}\|\mathbf{W}(\cdot s)\|_{L_{q_{1} / 2}(\Omega)}\right)^{p} d s\right)^{1 / p} .
\end{aligned}
$$

so that by the change of integration order and (56)

$\int_{2}^{T}\left(\langle t\rangle^{b-\frac{N}{2 q_{1}}} I_{q_{2}}(t)\right)^{p} d t \leq C\left(\frac{N}{2 q 2}\right)^{-\frac{p}{p^{\prime}}} \int_{2}^{T} d t \int_{t / 2}^{t-1}(t-s)-\left(\frac{N}{2 q_{2}}+1\right)\left(\langle s\rangle^{b}\|\mathbf{w}(\cdot s)\|_{L_{1} / 2}(\Omega)\right)^{p} d s$ $\leq C\left(\frac{N}{2 q 2}\right)^{-\frac{p}{p^{\prime}}} \int_{0}^{T}\left(\langle s\rangle^{b}\|\mathbf{w}(\cdot s)\|_{L_{q_{1} / 2}(\Omega)}\right)^{p} d s \int_{s+1}^{2 s}(t-s)^{-\left(\frac{N}{2 q_{2}}+1\right)} d t \leq C\left(\frac{N}{2 q_{2}}\right)-p\left(I+[\mathbf{v}]_{T}^{2}\right)$.

Analogously, by Holder's inequality

$$
\begin{aligned}
& <t>^{b-\frac{N}{2 q_{2}}} I I I_{q_{2}}(t) \leq C \int_{t-1}^{t}<s>^{b-\frac{N}{2 q_{2}}}\|\mathbf{w}(\cdot, s)\|_{L_{q_{2}}}(\Omega) d s, \\
& \leq C\left(\int_{t-1}^{t} d s\right)^{1 / p^{\prime}}\left(\int_{t-1}^{t}\left(<s>^{b}\|\mathbf{w}(\cdot, s)\|_{L_{q_{2}}(\Omega)}\right)^{p} d s\right)^{1 / p} \\
& \leq\left(\int_{t-1}^{t}\left(<s>^{b}\|\mathbf{w}(\cdot, s)\|_{L_{q_{2}}}(\Omega)^{p} d s\right)^{1 / p},\right.
\end{aligned}
$$

so that by the change of integration order and (56)

$\int_{2}^{T}\left(<t>^{b-\frac{N}{2 q_{2}}} I I I_{q_{2}}(t)\right)^{p} d t \leq C \int_{2}^{T} d t \int_{t-1}^{t}\left(<s>^{b}\|\mathbf{w}(\cdot, s)\|_{L_{q_{2}}(\Omega)}\right)^{p} d s$ $\leq C \int_{0}^{T}\left(<s>^{b}\|\mathbf{w}(\cdot, s)\|_{L_{q_{2}}(\Omega)}\right)^{p} d s \int_{s}^{s+1} d t \leq C\left(I+[\mathbf{v}]_{T}^{2}\right)$.

Summing up, we have obtained (68).

Recalling that $T \geq 2$, applying the maximal $\mathrm{L}_{\mathrm{p}}-\mathrm{L}_{\mathrm{q}}$ regularity theorem due to Shibata ${ }^{33}$ to Eq. (54)

and using (56) give that

$\left.\|\mathbf{u}\|_{L_{p}\left((0,2), H_{q}^{2}(\Omega)\right.}\right)+\left\|\partial_{t} \mathbf{u}\right\|_{L_{p}\left((0,2), L_{q}(\Omega)\right.} \leq C_{q}\left\|\ddot{e}_{0} \mathbf{w}\right\|_{L_{p}\left((0,2), L_{q}(\Omega)\right.} \leq C\left(I+[\mathbf{v}]_{T}^{2}\right)$

For any $q \in\left[q_{1} / 2, q_{2}\right]$. Employing the same argumentation as that in proving (29), by real interpolation,

we have

$$
\sup _{0<t<2}\|\mathbf{u}(\cdot, t)\|_{B_{q, p}^{2(1-1 / \mathrm{p})}(\Omega)} \leq C\left(I+[\mathbf{v}]_{T}^{2}\right)
$$

for any $q \in\left[q_{1} / 2, q_{2}\right]$. Combining (65), (66), (67), (68), (70), (71) and the Sobolev imbedding theorem, 
we have

$$
\begin{aligned}
& \left\|\langle t\rangle^{b} \mathbf{u}\right\|_{L_{p}\left((0, T) H_{\infty}^{1}(\Omega)\right)}+\|\left\langle t>^{\frac{N}{2 q_{1}}} \mathbf{u} \|_{L_{\infty}\left((0, T), L_{q_{1}}(\Omega)\right)}\right.
\end{aligned}
$$

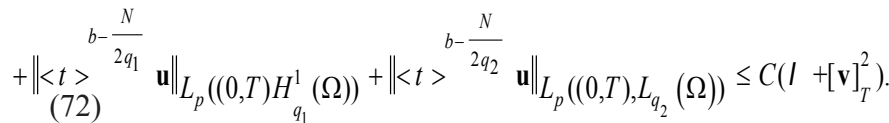

From (54), u satisfies the equations:

$$
\left\{\begin{array}{cl}
\partial_{t} \mathbf{u}+\lambda_{0} \mathrm{v}-J(T)^{-1} \operatorname{Div} \tilde{\mathbf{S}}(\mathbf{u}, \mathfrak{p})=-\lambda_{0} \mathrm{w}+\lambda_{0} \mathbf{u} & \text { in } \Omega \times(0, T), \\
\widetilde{\operatorname{div} \mathbf{u}}=0 & \text { in } \Omega \times(0, T), \\
\tilde{\mathbf{S}}(\mathbf{u}, \mathfrak{p})=0 & \text { on } \Gamma \times(0, T), \\
\left.\mathbf{u}\right|_{t=0}=0 & \text { in } \Omega
\end{array}\right.
$$

so that by Theorem 8 ,

$$
\begin{aligned}
& \left\|<t>^{b-\frac{N}{2 q_{2}}} \mathbf{u}\right\|_{L_{p}\left((0, T) H_{q_{2}}^{2}(\Omega)\right)}+\left\|<t>^{b-\frac{N}{2 q_{2}}} \partial_{t} \mathbf{u}\right\|_{L_{p}}\left((0, T), L_{q_{2}}(\Omega)\right) \\
& +C\left\|<t>^{b-\frac{N}{2 q_{2}}}(\mathbf{u}, \mathbf{w})\right\|_{L_{p}}\left((0, T) L_{q_{2}}(\Omega)\right),
\end{aligned}
$$

which, combined with (72), furnishes that

$$
[\mathbf{u}]_{T} \leq C\left(I+[\mathbf{v}]_{T}^{2}\right)
$$

Since $\mathrm{v}=\mathbf{w}+\mathbf{u}$, by (58) and (73), we see that $\mathrm{v}$ satisfies the inequality (50), which completes the proof of Theorem $1 .{ }^{53-56}$

\section{Acknowledgments}

None.

\section{Conflicts of interest}

Author declares that there is no conflict of interest.

\section{References}

1. Solonnikov VA. Solvability of the problem of evolution of an isolated amount of a viscous incom-pressible capillary fluid. Zap Nauchn Sem Leningrad Otdel mat Inst Steklov LOMI. 1984;140:179-186.

2. Solonnikov VA. On nonstationary motion of a finite isolated mass of selfgravitating fluid. Algebra I Analiz. 1990;1(1):207-249.

3. Solonnikov VA. Solvability of the problem of evolution of a viscous incompressible fluid bouded by a free surface on a finite time interval. Algebra i Analiz. 1991;3(1):222-257.

4. Solonnikov VA. Lectures on evolution free boundary problems: classical solutions. Mathematical aspects of evolving interfaces, Lecture Notes in Math. 2003;1812:123-175.

5. Schweizer B. Free boundary fluid systems in a semigroup approach and oscillatory behavior. SIAM J Math Anal. 1997;28(5):1135-1157.

6. Moglievskii SH, Solonnikov VA. On the solvability of a free boundary problem for the Navier-Stokes equations in the Holder space of functions. Nonlinear Analysis Sc Norm Super di Pisa Quaderni Scuola Norm. 1991. p. 257-271.

7. Solonnikov VA. On the transient motion of an isolated volume of viscous incompressible fluid. Math. USSR Izvestiya. 1988;31(2):381-405.
8. Mucha PB, Zajaczkowski W. On the existence for the Cauchy-Neumann problem for the Stokes system in the $\mathrm{L}_{\mathrm{p}}$-framework. Studia Math. 2000;143(1):75-101.

9. Mucha PB, Zajaczkowski W. On local existence of solutions of the free boundary problem for an incompressible viscous self-gravitating fluid motion. Appl Math. 2000;27(3):319-333.

10. Shibata Y, Shimizu S. On a free boundary problem for the Navier-Stokes equations. Di $\square$ erential Integral Equations. 2007;20(3):241-276.

11. Shibata $\mathrm{Y}$, Shimizu S. On the $\mathrm{L}_{\mathrm{p}}-\mathrm{L}_{\mathrm{q}}$ maximal regularity of the Neumann problem for the Stokes equations in a bounded domain. J Reine Angew Math. 2008;61:157-209.

12. Shibata Y. On some free boundary problem of the Navier-Stokes equations in the maximal $\mathrm{L}_{\mathrm{p}}-\mathrm{L}_{\mathrm{q}}$ regularity class. $J$ Di $\square$ erential Equations. 2015;258(12):4127-4155.

13. Solonnikov VA. Unsteady flow of a finite mass of a fluid bounded by a free surface. Zap Nauchn Sem Leningrad Otdel mat Inst Steklov LOMI. $1986 ; 152: 137-157$

14. Padula M, Solonnikov VA. On the global existence of nonsteady motion of a fluid drop and their exponential decay to a uniform rigid rotation. Ouad Mat. 2002;10:185-218.

15. Shibata Y. Global well-posedness of unsteady motion of viscous incompressible capillary liquid bounded by a free surface. 2002.

16. Allain G. Small-time existence for Navier-Stokes equations with a free surface. Appl Math Optim. 1987;16(1):37-50.

17. Beale JT. The initial value problem for the Navier-Stokes equations with a free surface. Commun Pure Appl Math. 1981;34(3):359-392.

18. Tani A. Small-time existence for the three-dimensional Navier-Stokes equations for an incompressible fluid with a free surface. Arch Rational Mech Anal. 1996;133(4):299-331.

19. Abels $\mathrm{H}$. The initial-value problem for the Navier-Stokes equations with a free surface in Lq-Sobolev spaces. Adv Differential Equations. 2005;10(1):45-64.

20. Beale T. Large-time regularity of viscous surface waves. Arch Rational Mech Anal. 1984;84(4):307-352.

21. Tani I, Tanaka N. Large-time existence of surface waves in incompressible viscous fluids with or without surface tension. Arch Rational Mech anal. 1995;130(4):303-314.

22. Donna Lynn, Gates Sylvester. Large time existence of small viscous surface waves without surface tension. Commun. Partial Differential Equations. 1990;15(6):823-903.

23. Beale JT, Nishida T. Large-time behaviour of viscous surface waves Recent topics in nonlinear PDE, North-Holland Math. Stud, Amsterdam, Netherlands; 1985. p. 1-14.

24. Sylvester DLG. Decay rate for a two-dimensional viscous ocean of finite depth. J Math Anal Appl. 1996;202(0340):659-666.

25. Hataya Y. Decaying solution of a Navier-Stokes flow without surface tension. J Math Kyoto Univ. 2009;49(4):691-717.

26. Hataya Y, Kawashima S. Decaying solution of the Navier-Stokes flow of infinite volume without surface tension. Nonlinear Analysis. $2009 ; 71: 2535-2539$

27. Saito H, Shibata Y. On decay properties of solutions to the Stokes equations with surface tension and gravity in the half space. J Math Soc Japan. 2016;68(4):1559-1614

28. Saito H, Shibata Y. Global existence and large-time behavior of solutions to the Navier-Stokes equations with a free surface. 2002. 
29. Shibata Y. Local well-posedness of free surface problems for the Navier-Stokes equations in a general domain. Discrete and Continuous Dynamical Systems Series S. 2016;9(1):315-342.

30. Shibata Y. Local well-posedness for the free boundary problem of the Navier-Stokes equations in an exterior domain. 2002.

31. Abels H. On generalized solutions of two-phase flows for viscous incompressible fluids. Interfaces Free Bound. 2007;9(1):31-65.

32. Denisova V. A priori estimates for the solution of the linear nonstationary problem connected with the motion of a drop in a liquid medium. Proc Steklov Inst Math. 1991;188:1-24.

33. Denisova V. Problem of the motion of two viscous incompressible fluids separated by a closed free interface. Acta Appl Math. 1994;37(1-2):31-40.

34. Denisova V, Solonnikov VA. Classical solvability of the problem on the motion of two viscous incompressible fluids. St. Petersburg Math. 1996;7(5):755-786.

35. Giga Y, Takahashi SH. On global weak solutions of the nonstationary two-phase Stokes flow. SIAM J Math Anal. 1994;25(3):876-893.

36. Köhne M, Pruss J, Wilke M. Qualitative behavior of solutions for the two-phase Navier-Stokes equations with surface tension. Math Ann. 2013;356(2):737-792.

37. Maryani $\mathrm{S}$, Saito $\mathrm{H}$. On the $R$-boundedness of solution operator families for two-phase Stokes resolvent equations. Diff Int Eqs. 2017;30(1/2):1-52.

38. Nouri A, Poupaud F. An existence theorem for the multifluid NavierStokes problem. J Deferential Equations. 1995;122:71-88.

39. Pruess J, Simonett G. On the two-phase Navier-Stokes equations with surface tension. Interfaces and Free Boundaries. 2010;12(3):311-345.

40. Pruess J, Simonett G. Analytic solutions for the two-phase NavierStokes equations with surface tension and gravity, Progress in Nonlinear Differential Equations and Their Applications. 2011;80:507-540.

41. Pruess J, Simonett G. Moving Interfaces and Quasilinear Parabolic Evolution Equations. Birkhauser Monographs in Mathematics. USA: Springer International Publishing; 2016. 609 p.

42. Shibata Y, Shimizu S. On the resolvent estimate of the interface problem for the Stokes system in a bounded domain. J Differential Equations. 2003;191:408-444.

43. Shibata $\mathrm{Y}$, Shimizu S. Maximal $\mathrm{L}_{\mathrm{p}}-\mathrm{L}_{\mathrm{q}}$ regularity for the two-phase Stokes equations; Model problems. $J$ Differential Equations. 2011;251(2):373-419.
44. Simonett G, Wilke V. Stability of equilibrium shapes in some free boundary problems involving fluids. Handbook of Mathematical Analysis in Mechanics Viscous Fluids. 2017:1-46.

45. Takahashi SH. On global weak solutions of the nonstationary two-phase Navier-Stokes flow. Adv Math Sci Appl. 1995;5(1):321-342.

46. Tanaka N. Two-phase free boundary problem for viscous incompressible thermocapillary convection. Japan JMath (New Series). 1995;21(1):1-42.

47. Shibata Y. On $L_{p}-L_{q}$ decay estimate for Stokes equations with free boudary condition in an exterior domain. 2001.

48. Shibata Y, Shimizu S. Decay properties of the Stokes semigroup in exterior domains with Neumann boundary condition. J Math Soc Japan. 2007;59(1):1-34

49. Shibata Y. On the R-boundedness of solution operators for the Stokes equations with free boundary condition. Diff Int Eqns. 2014;27(3/4):313-368

50. Shibata Y. On the R-bounded solution opeator and the maximal $L_{p}$ $\mathrm{L}_{\mathrm{g}}$ regularity of the Stokes equations with free boundary condition Mathamtical Fluid Dynamics, Present and Future. 2016;183:203-285.

51. Tanabe H. Functional Analytic Methods for Parital Differential Equations. Pure and Applied Mathematics. New York: A Series of Monographs and Textbooks, Marcel Dekker; 1997. 414 p.

52. Meyries M, Schnaubelt R. Interpolation, embeddings and traces of anisotropic fractional Sobolev spaces with temporal weights. J Func Anal. 2012;262:1200-1229.

53. Shibata Y, Shimizu S. On the maximal $\mathrm{L}_{\mathrm{p}}-\mathrm{L}_{\mathrm{q}}$ regularity of the Stokes problem with first order boundary condition; model problems. $J$ Math Soc Japan. 2012;64(2):561-626.

54. Shimizu S. Local solvability of free boundary problems for two-phase Navier-Stokes equations with surface tension in the whole space. Progr Nonlinear Differential Equations Appl. 2011;80:647-686.

55. Solonnikov VA. Estimates of solutions of an initial-boundary value problem for the linear non-stationary Navier-Stokes system. Zap Nauchn Sem Leningrad Otdel mat Inst Steklov LOMI. 1976;59:178-254.

56. Solonnikov VA. Unsteady flow of a finite mass of a fluid bounded by a free surface. Zap Nauchn Sem Leningrad Otdel mat Inst Steklov LOMI. 1986; $152: 137-157$ 